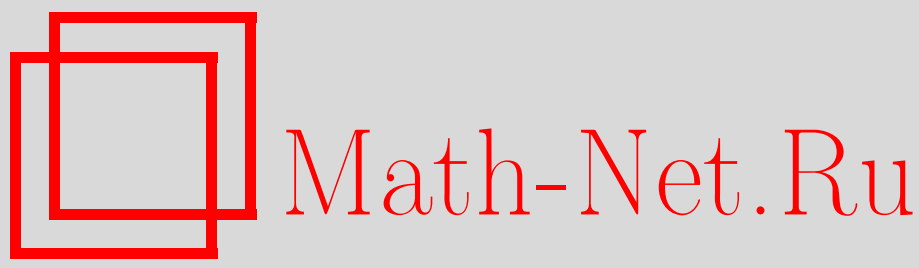

С. А. Назаров, А. С. Слуцкий, Одномерные уравнения деформации тонких слабоискривленных стержней. Асимптотический анализ и обоснование, Изв. РАН. Сер. матем., 2000, том 64, выпуск 3, 97-130

DOI: https://doi.org/10.4213/im290

Использование Общероссийского математического портала Math-Net.Ru подразумевает, что вы прочитали и согласны с пользовательским соглашением

http://www.mathnet.ru/rus/agreement

Параметры загрузки:

IP : 3.89 .185 .249

26 апреля 2023 г., 16:54:09 
УДК 517.946

\author{
С. А. Назаров, А.С. Слуцкий
}

\title{
Одномерные уравнения деформации тонких слабоискривленных стержней. Асимптотический анализ и обоснование
}

\begin{abstract}
Строится асимптотика решения пространственной задачи теории упругости в тонком теле (стержне) с плавно изменяющимся сечением. Допускается произвольная анизотропия и неоднородность материала. Торцы стержня, находящегося под действием объемных сил, жестко защемлены, а к боковой поверхности приложены усилия. Малым параметром $h$ служит отношение длины стержня к максимальному его диаметру. Указаны требования к дифференциальным свойствам и структуре внешних нагрузок, при которых решение одномерных уравнений, появляющихся в результате асимптотического анализа, доставляет приемлемое приближение к трехмерным полям смещений и напряжений. Оценка погрешности базируется на специальном неравенстве Корна, асимптотическая точность которого достигается распределением весовых множителей и степеней $h$ в $L_{2}$-нормах смещений и их производных.
\end{abstract}

Библиограффия: 39 наименований.

\section{§ 1. Постановка задачи и предварительное описание результатов}

1. Задача теории упругости. Пусть $\omega$ - область на плоскости $\mathbb{R}^{2}$, ограниченная простым замкнутым контуром $\partial \omega$, и $\varkappa$ - гладко зависящий от параметра $z \in[-1,1]$ диффеоморфизм: $\mathbb{R}^{2} \rightarrow \mathbb{R}^{2}$. Положим $\omega(z)=\varkappa_{z} \omega$ и определим тонкий криволинейный стержень формулой

$$
\Omega_{h}=\left\{x=(y, z) \in \mathbb{R}^{3}:|z|<1, \eta=h^{-1} y \in \omega(z)\right\}
$$

Здесь $h>0$ - малый параметр, $y=\left(y_{1}, y_{2}\right)$ и $\eta=\left(\eta_{1}, \eta_{2}\right)$ - "медленные" и "быстрые" переменные в сечениях стержня (поскольку длина стержня равна двум, величины $y_{i}, \eta_{i}$ и $h$ безразмерные).

Рассмотрим линейную задачу теории упругости, допуская произвольную анизотропию и неоднородность материала. Воспользуемся матричной записью уравнений; при этом вектор смешений $u=\left(u_{1}, u_{2}, u_{3}\right)^{t}$ интерпретируем как столбец $(t-$ знак транспонирования). Введем еще столбец деформаций

$$
\varepsilon(u)=\left(\varepsilon_{11}, \varepsilon_{22}, \varepsilon_{33}, \alpha^{-1} \varepsilon_{32}, \alpha^{-1} \varepsilon_{31}, \alpha^{-1} \varepsilon_{13}\right)^{t}
$$


и аналогичный столбец напряжений $\sigma(u)$, записывая закон Гука в виде $\sigma(u)=$ $A \varepsilon(u)$. Здесь $\varepsilon=\left(\varepsilon_{i j}\right)$ - тензор деформаций с декартовыми компонентами

$$
\varepsilon_{i j}=\frac{1}{2}\left(\frac{\partial u_{j}}{\partial x_{k}}+\frac{\partial u_{k}}{\partial x_{j}}\right),
$$

$A$ - симметрическая положительно определенная матрица-функция, составленная из упругих модулей материала и гладко зависящая от переменных $\eta$ и $z$. Множитель $\alpha^{-1}=2^{1 / 2}$ введен в (1.2) для того, чтобы совпали естественные нормы тензора и столбца деформаций (напряжений). Согласно (1.2) и (1.3)

$$
\varepsilon(u)=D\left(\nabla_{x}\right)^{t} u,
$$

где $\nabla_{x}=\operatorname{grad}$ и $D-(3 \times 6)$-матрица дифференциальных операторов,

$$
D(\xi)=\left(\begin{array}{cccccc}
\xi_{1} & 0 & 0 & 0 & \alpha \xi_{3} & \alpha \xi_{2} \\
0 & \xi_{2} & 0 & \alpha \xi_{3} & 0 & \alpha \xi_{1} \\
0 & 0 & \xi_{3} & \alpha \xi_{2} & \alpha \xi_{1} & 0
\end{array}\right), \quad \alpha=\frac{1}{\sqrt{2}} .
$$

Непосредственной проверкой можно убедиться, что уравнения теории упругости принимают вид

$$
\begin{gathered}
D\left(-\nabla_{x}\right) A\left(h^{-1} y, z\right) D\left(\nabla_{x}\right)^{t} u(h, x)=f(h, x), \quad x \in \Omega_{h}, \\
D(n(h, x)) A\left(h^{-1} y, z\right) D\left(\nabla_{x}\right)^{t} u(h, x)=g(h, x), \quad x \in \Gamma_{h}, \\
u(h, x)=0, \quad x \in \omega_{h}( \pm 1) .
\end{gathered}
$$

Они означают, что на стержень действуют объемные силы $f=\left(f_{1}, f_{2}, f_{3}\right)^{t}, \mathrm{k}$ боковой поверхности $\Gamma_{h}=\left\{x \in \partial \Omega_{h}:|z|<1\right\}$ приложены усилия $g=\left(g_{1}, g_{2}, g_{3}\right)^{t}$, а торцы стержня $\omega_{h}( \pm 1)=\{x: \eta \in \omega( \pm 1), z= \pm 1\}$ жестко зашемлены. В (1.7) $n=\left(n_{1}, n_{2}, n_{3}\right)^{t}$ - единичный вектор внешней нормали к $S_{h}$. Дифференциальные операторы, фигурирующие в (1.6) и (1.7), обозначаем $L\left(h, x, \nabla_{x}\right)$ и $B\left(h, x, \nabla_{x}\right)$.

Как обычно, под обобщенным решением задачи (1.6)-(1.8) в пространстве Соболева $H^{1}\left(\Omega_{h}\right)^{3}$ (тройка указывает количество компонент вектор-функций) понимается элемент $u$ подпространства $\stackrel{\circ}{H}^{1}\left(\Omega_{h}\right)^{3}=\left\{u \in H^{1}\left(\Omega_{h}\right)^{3}: u=0\right.$ на $\left.\omega_{h}( \pm 1)\right\}$, удовлетворяюший при любой пробной функции $v \in \stackrel{\circ}{H^{1}}\left(\Omega_{h}\right)^{3}$ интегральному тождеству

$$
E\left(u, v ; \Omega_{h}\right)=(f, v)_{\Omega_{h}}+(g, v)_{\Gamma_{h}} .
$$

Здесь $(\cdot, \cdot) \Xi-$ скалярное произведение в $L_{2}(\Xi), 2^{-1} E\left(u, u ; \Omega_{h}\right)$ - функционал упругой энергии, запасенной телом $\Omega_{h}$,

$$
E(u, v ; \Xi)=\left(A D\left(\nabla_{x}\right)^{t} u, D\left(\nabla_{x}\right) v\right)_{\Xi} .
$$

Отметим, что тождество (1.9) возникает при учете формулы Грина

$$
(L u, v)_{\Omega_{h}}+(B u, v)_{\Gamma_{h}}=E\left(u, v ; \Omega_{h}\right),
$$

справедливой, например, для $u \in H^{2}\left(\Omega_{h}\right)^{3}$ и $v \in \stackrel{\circ}{H^{1}}\left(\Omega_{h}\right)^{3}$.

Следуюшее утверждение о разрешимости задачи (1.6)-(1.8) (или (1.9)) известно. 
ПРЕДЛОЖЕНИЕ 1.1. Если $f \in L_{2}\left(\Omega_{h}\right)^{3}, g \in L_{2}\left(\Gamma_{h}\right)^{3}$, то существует единственное обобщенное решение $u \in \stackrel{\circ}{H}^{1}\left(\Omega_{h}\right)^{3}$ задачи (1.6)-(1.8) и справедлива оченка

$$
\left\|u ; H^{1}\left(\Omega_{h}\right)\right\| \leqslant c_{h}\left(\left\|f ; L_{2}\left(\Omega_{h}\right)\right\|+\left\|g ; L_{2}\left(\Gamma_{h}\right)\right\|\right) .
$$

Подчеркнем, что постоянная $c_{h}$ сушественно зависит от малого параметра $h$ (но не от $f, g$ и $u$ ). Выявление и спецификация этой зависимости составляет предмет $\S 3$ статьи, где отыскивается правильная запись весового неравенства Корна, учитываюшая геометрию тонкого тела $\Omega_{h}$. Однако основная цель работы - построение и оправдание асимптотики решения задачи (1.6)-(1.8) при $h \rightarrow+0$.

2. Описание результатов. Теория тонких стержней, являющаяся одной из важных прикладных дисциплин, имеет давнюю историю. Прежде всего нужно упомянуть так называемые технические теории изгиба стержней. В них упрощение трехмерных уравнений теории упругости и формулировки одномерных моделей основываются на ряде физических допущений (гипотез), а цели и методика исследований не подразумевают какого-либо математического обоснования. Основы современной теории стержней заложены А. Клебшем [1]. Дальнейшее развитие теория стержней получила в трудах [2], [3] и др. К настояшему времени технической теории изгиба стержней изотропных и анизотропных, прямых и искривленных посвящено огромное количество работ (упомянем монографии [4]-[8], статьи [9], [10]). Технические теории многие десятилетия используются в инженерных расчетах, но вопросы об условиях их применимости, обеспечиваемой точности приближения и, вообще, об адекватности одномерных моделей реальным объектам остаются открытыми до сих пор.

Явное вхождение малого параметра в задачи о стержнях, пластинах и оболочках делает их привлекательными для математиков, и к настояшему времени разработано множество подходов к асимптотическому анализу задач теории упругости для тонких тел, в частности для стержней. Во-первых, имеется ряд публикаций, посвященных формальным асимптотическим конструкциям (см. [11]-[14] и др.); в них предположение о справедливости того или иного анзаца, т.е. асимптотической структуры решения, в целом играет ту же роль, что и упоминавшиеся физические гипотезы, а существо математического исследования состоит в описании процедуры построения элементов анзаца и проверке разрешимости (результирующей) задачи на подмногообразии меньшей размерности, к которому стягивается тонкий объект (ср. с $\S 2$ данной статьи). Во-вторых, саму идею редукции размерности объясняет метод доказательства теорем о сходимости в подходящем смысле решений трехмерных задач к решениям результируюших задач (см. [15], [16] для пластин и [17]-[22] для стержней; отметим, что во всех этих публикациях речь идет об изотропных тонких телах). Однако более точная информация о поведении решения при $h \rightarrow+0$ (в том числе и скорость сходимости) зачастую остается за рамками такого подхода, и потому сформулированные выше вопросы, важные для практических целей, требуют продолжения исследований. В-третьих, разработана методика построения полных асимптотических разложений, включающая 
изучение явления пограничного слоя около торцов стрежней (см. [23]-[26] и др., а также $[27$, гл. 15,16$])$. Именно, при условии бесконечной дифференцируемости данных задачи и с помошью решения рекуррентной последовательности предельных задач члены асимптотического ряда определяются так, что путем удлинения его частичной суммы удается невязки, порожденные ею в исходных уравнениях, сделать сколь угодно малыми. Преимушество подобной методики (возможность обоснования асимптотики при наличии какой-нибудь, даже очень грубой, априорной оценки решения) оборачивается и ее недостатком, т.е. из-за завышенных требований к правым частям без ответа остается основной вопрос: какие же их свойства обеспечивают возможность редукции размерности? Вместе с тем, она (методика) обеспечивает наиболее полное представление о свойствах решения при малых значениях геометрического параметра $h$, хотя младшие члены асимптотики вряд ли могут быть востребованы для приложений.

Настояшая работа посвяшена изучению главного члена асимптотики решения задачи (1.6)-(1.8) и определению условий на правые части $f$ и $g$, достаточных для справедливости неулучшаемой (по показателю степени $h$ ) оценки разности истинного и асимптотического решений. В $§ 2$ приводятся используемые асимптотические конструкции. Процедура их построения следует общей схеме из [23], [28], [29] (см. также [27, гл. 16]), но включает модификации, связанные с вариацией сечения стержня (ср. с [30]). В силу матричной формы записи дифференциальных операторов (см. (1.6) и (1.7)) выкладки, формирующие результирующие задачи, сведены к необходимому минимуму; кроме того, они допускают произвольные анизотропию и неоднородность материала и форму сечения. Единственное ограничение геометрического плана имеет чисто технический характер и возникает в $\S 3$ при выводе неравенства Корна, приспособленного к геометрии тонкого искривленного стержня (1.1). Известно (см. [7], [31], [32] и др.), что асимптотическая точность такого неравенства обеспечивается добавлением весовых множителей и степеней малого параметра $h$ в нормы смешений и их производных; при этом необходимо различать направления поперек и вдоль оси стержня. Весовое неравенство Корна, установленное в теореме 3.1 , дает возможность в $\S 4$ обосновать главньй член $\mathbf{U}$ асимптотики решения $u$ задачи (1.6)-(1.8). Именно, в теореме 4.3 содержится такая оценка:

$$
|u-\mathbf{U}| \leqslant c_{\delta} h^{(1-\delta) / 2}\left(\mathscr{N}_{0}+\widetilde{\mathscr{N}}\right)
$$

здесь $\delta$ - произвольное положительное число, а | · - норма, приспособленная к асимптотической структуре решения (см. определение (3.4) и п. $2 \S 3$, где поясняется его правильность). Особенно важным является то, что величины $\mathscr{N}_{0}$ и $\widetilde{\mathscr{N}}$, указанные в (4.13) и (4.24), вбирают в себя всю зависимость мажоранты в оценке (1.13) от правых частей $f$ и $g$, т.е. постоянная $c_{\delta}$ зависит от числа $\delta$, формы стержня и упругих свойств материала, но не меняется при допустимом варьировании нагрузок. Тем самым неравенство (1.13) указывает требования к структуре $f, g$ и к дифференциальным свойствам ее элементов, обеспечивающие редукцию размерности в задаче о стержне. Оптимальность этих требований обсуждается в п. $7 \S 2$ и п. $4 \S 4$, где, в частности, высказывается гипотеза о необходимых условиях. 
3. Асимптотический анзац. В [29] изучен широкий класс краевых задач в тонких областях, выделяемый так называемым полиномиальным свойством. Иными словами, квадратичная форма вида (1.10) должна вырождаться только на конечномерном линеале $\mathscr{R}$ векторных полиномов. Как вытекает из (1.4), (1.5) и (1.10), в рассматриваемой задаче теории упругости этот линеал

$$
\mathscr{R}=\left\{u: u(x)=a+b \times x, a, b \in \mathbb{R}^{3}\right\}
$$

состоит из смещений тела как жесткого целого (в (1.14) крестом обозначено векторное произведение). Если линеал $\mathscr{R}$ известен, то согласно [29] определение размеров результируюшей системы и порядков ее элементов, проверка эллиптичности и разрешимости соответствуюшей задачи Дирихле сводятся к несложным алгебраическим операциям. Так, в случае редукции размерности к единице достаточно указать в $\mathscr{R}$ базис $\left\{p^{j, k} \mid j=1, \ldots, J, k=0, \ldots, \tau_{j}-1\right\}$, подчиненньй условиям

$$
\partial_{z} p^{j, k}(y, z)=p^{j, k-1}(y, z), \quad k=0, \ldots, \tau_{j}-1,
$$

где $p^{j,-1}=0$. Для линеала жестких смещений (1.14) нужньй базис вьглядит следующим образом:

$$
\begin{gathered}
p^{i, 0}=e^{i}, \quad p^{i, 1}(y, z)=e^{i} z-e^{3} y_{i}, \quad i=1,2, \\
p^{3,0}=e^{3}, \quad p^{4,0}(y)=\alpha\left(y_{1} e^{2}-y_{2} e^{1}\right)
\end{gathered}
$$

$\left(e^{j}\right.$ - орт оси $\left.x_{j}, j=1,2,3\right)$. При этом $J=4$ и $\tau_{1}=\tau_{2}=2, \tau_{3}=\tau_{4}=1$. Общие результаты [29] обслуживают случай прямолинейного однородного стержня и утверждают, что редукция размерности в задаче (1.6)-(1.8) приводит к эллиптической системе обыкновенных дифференциальных уравнений с оператором $\mathbf{M}\left(\partial_{z}\right)=\left(\mathbf{M}_{p q}\left(\partial_{z}\right)\right)_{p, q=1}^{J}$, в котором ord $\mathbf{M}_{p q}=\tau_{p}+\tau_{q}$. В $\S 2$, приспосабливая процедуру построения асимптотики к тонкой искривленной области (1.1), мы обнаруживаем результирующую систему (2.36) с оператором $\mathbf{M}\left(z, \partial_{z}\right)$ той же структуры (разумеется, у него переменные коэффициенты). Кроме того, мы пользуемся начальными членами асимптотического анзаца, найденного в работе [29] для общих краевых задач с полиномиальным свойством:

$$
\begin{aligned}
& \sum_{j=1}^{J} h^{-\tau_{j}} \sum_{q=0}^{-\tau_{j}-1} h^{q} p^{q, j}(\eta, 0) \frac{\partial^{q} w_{j}}{\partial z^{q}}(z)=h^{-2}\left\{e^{1} w_{1}(z)+e^{2} w_{2}(z)\right\} \\
& \quad+h^{-1}\left\{e^{3}\left(w_{3}(z)-\eta_{1} \partial_{z} w_{1}(z)-\eta_{2} \partial_{z} w_{2}(z)\right)+\alpha\left(\eta_{1} e^{2}-\eta_{2} e^{1}\right) w_{4}(z)\right\},
\end{aligned}
$$

где $w_{1}, w_{2}, w_{3}$ и $w_{4}$ - функции, подлежащие определению (соответственно продольные и поперечное смещения в стрежне и его закручивание). Отметим, что искривленность стрежня не влияет на члены асимптотики, выделенные в (1.15), а для вычисления всего оператора $\mathbf{M}\left(z, \partial_{z}\right)$ достаточно построить лишь очередной асимптотический член $h^{0} U^{0}(\eta, z)$ (см. п. $6 \S 2$ ). 
Расхождение показателей степеней $h$ при $w_{q}$ имеет простое механическое истолкование: стержень легче изогнуть, чем растянуть или скрутить. Подчеркнем, что серьезное (см. замечание 2.3) отклонение от анзаца (1.15) приводит к необходимости сузить постановку задачи (1.6)-(1.8); например, в [26] ограничения на симметрию физических и геометрических характеристик стержня возникли именно из-за неудачно выбранной структуры асимптотического ряда.

\section{$\S 2$. Формальная схема построения асимптотики}

1. Вспомогательные конструкции. Замена координат $x \mapsto(\eta, z)$ влечет расщепление оператора (1.5):

$$
D\left(\nabla_{x}\right)=h^{-1} D\left(\nabla_{\eta}, 0\right)+D\left(0, \partial_{z}\right) \equiv h^{-1} \mathbb{D}_{\eta}+\mathbb{D}_{z},
$$

здесь $\partial_{z}=\partial / \partial z$. Нормаль $n$ к боковой поверхности $\Gamma_{h}$ стержня $\Omega_{h} \subset \mathbb{R}^{3}$ удовлетворяет соотношению

$$
N(h, x)^{1 / 2} n(h, x)=\left(\nu_{1}(\eta, z), \nu_{2}(\eta, z), h \nu_{0}(\eta, z)\right)^{t},
$$

в котором $\nu^{t}=\left(\nu_{1}, \nu_{2}\right)^{t}$ - единичный вектор внешней нормали к гранище области $\omega(z) \subset \mathbb{R}^{2}$ и $N(h, x)=1+h^{2} \nu_{0}(\eta, z)^{2}$. Таким образом,

$$
N^{1 / 2} D(n)=D(\nu, 0)+h \nu_{0} D(0,1) \equiv \mathbb{D}_{\nu}+h \nu_{0} \mathbb{D}_{1},
$$

причем в $(2.1) \mathbb{D}_{z}=\mathbb{D}_{1} \partial_{z}$. Операторы $L$ и $B$ из (1.6) и (1.7) допускают расщепления

$$
\begin{aligned}
L\left(h, x, \nabla_{x}\right)= & h^{-2} L^{0}\left(\eta, z, \nabla_{\eta}\right) \\
& +h^{-1} L^{1}\left(\eta, z, \nabla_{\eta}, \partial_{z}\right)+h^{0} L^{2}\left(\eta, z, \partial_{z}\right), \\
N(h, x)^{1 / 2} B\left(h, x, \nabla_{x}\right)= & h^{-1} B^{0}\left(\eta, z, \nabla_{\eta}\right) \\
& +h^{0} B^{1}\left(\eta, z, \nabla_{\eta}, \partial_{z}\right)+h^{1} B^{2}\left(\eta, z, \partial_{z}\right)
\end{aligned}
$$

с коэффициентами

$$
\begin{array}{lll}
L^{0}=-\mathbb{D}_{\eta} A \mathbb{D}_{\eta}^{t}, & L^{1}=-\mathbb{D}_{\eta} A \mathbb{D}_{z}^{t}-\mathbb{D}_{z} A \mathbb{D}_{\eta}^{t}, & L^{2}=-\mathbb{D}_{z} A \mathbb{D}_{z}^{t}, \\
B^{0}=\mathbb{D}_{\nu} A \mathbb{D}_{\eta}^{t}, & B^{1}=\mathbb{D}_{\nu} A \mathbb{D}_{z}^{t}+\nu_{0} \mathbb{D}_{1} A \mathbb{D}_{\eta}^{t}, & B^{2}=\nu_{0} \mathbb{D}_{1} A \mathbb{D}_{z}^{t}
\end{array}
$$

Правые части $f$ и $g$ задачи (1.6)-(1.8) запишем в форме

$$
\begin{aligned}
& f(h, x)=h^{-1} f^{0}(\eta, z)+h^{0} \bar{f}^{0}(z)+\tilde{f}(h, x), \\
& g(h, x)=h^{-1} g^{0}(\eta, z)+\tilde{g}(h, x),
\end{aligned}
$$


причем остатки $\tilde{f}$ и $\tilde{g}$ считаем малыми (для уточнения см. $\S 4$ ), а выделенные члены подчиним условиям

$$
\begin{gathered}
\left(f^{0}, e^{i}\right)_{\omega(z)}+\left(g^{0}, e^{i}\right)_{\partial \omega(z)}=0, \quad i=1,2, \\
f_{3}^{0}(z)=0
\end{gathered}
$$

где $z \in(-1,1), e^{i}$ - орт оси $O x_{i}$. Задача (1.6)-(1.8) линейна, и потому требования (2.6)-(2.8) всегда можно соблюсти за счет введения нормируюших множителей и нулевых слагаемых. Представления (2.6), полностью согласованные с анзацем (1.15), кажутся искусственными только на первый взгляд - в п. 7 обсуждается их механическая интерпретация.

Вспоминая сказанное в п. $3 \S 1$ и учитывая $(2.4),(2.6)$, предлагаем такое асимптотическое представление решения:

$$
\begin{aligned}
u(h, x)= & h^{-2} \mathscr{U}^{(-2)}(z)+h^{-1} \mathscr{U}^{(-1)}(\eta, z)+h^{0} \mathscr{U}^{(0)}(\eta, z) \\
& +h^{1} \mathscr{U}^{(1)}(\eta, z)+h^{2} \mathscr{U}^{(2)}(\eta, z)+\cdots
\end{aligned}
$$

Подставляя $(2.4)$ и $(2.6),(2.9)$ в (1.6), (1.7) и собирая коэффициенты при одинаковых степенях малого параметра $h$, приходим к набору двумерных задач на сечении $\omega(z)$ :

$$
\begin{aligned}
& L^{0} \mathscr{U}^{(j)}=-L^{1} \mathscr{U}^{(j-1)}-L^{2} \mathscr{U}^{(j-2)}+\delta_{j, 1} f^{0} \text { в } \omega(z), \\
& B^{0} \mathscr{U}^{(j)}=-B^{1} \mathscr{U}^{(j-1)}-B^{2} \mathscr{U}^{(j-2)}+\delta_{j, 1} g^{0} \text { на } \partial \omega(z),
\end{aligned}
$$

здесь $j=-2,-1,0,1$ и $\mathscr{U}^{(p)}=0$ при $p<-2$. Отметим, что при выводе (2.11) использовано очевидное соотношение $N(h, x)^{1 / 2}=1+O\left(h^{2}\right)$. Следуюшее утверждение известно (или легко проверяемо).

ПрЕДЛОЖЕНИЕ 2.1. Пусть $(\mathscr{F}, \mathscr{G}) \in H^{l-1}(\omega(z))^{3} \times H^{l-1 / 2}(\partial \omega(z))^{3} u l \in \mathbb{N}=$ $\{1,2, \ldots\}$. Краевая задача

$$
L^{0} \mathscr{U}=\mathscr{F} \quad \boldsymbol{\theta} \quad \omega(z), \quad B^{0} \mathscr{U}=\mathscr{G} \quad \text { на } \quad \partial \omega(z)
$$

имеет решение $\mathscr{U} \in H^{l+1}(\omega(z))^{3}$ в том и только том случае, если выполнены условия

$$
\left(\mathscr{F}, \Phi^{q}\right)_{\omega(z)}+\left(\mathscr{G}, \Phi^{q}\right)_{\partial \omega(z)}=0, \quad q=1, \ldots, 4
$$

əде

$$
\Phi^{1}=e^{1}, \quad \Phi^{2}=e^{2}, \quad \Phi^{3}=e^{3}, \quad \Phi^{4}=\alpha\left(\eta_{1} e^{2}-\eta_{2} e^{1}\right) .
$$

Решение определено с точностью до линейной комбиначии векторов (2.14), а решение, нормированное условиями

$$
\left(\mathscr{U}, \Phi^{q}\right)_{\omega(z)}=0, \quad q=1, \ldots, 4,
$$

становится единственным, наследует от правых частей гладкость относительно параметра $z \in[-1,1]$ и подчиняется оченке

$$
\left\|\mathscr{U} ; H^{l+1}(\omega(z))\right\| \leqslant c\left\|(\mathscr{F}, \mathscr{G}) ; H^{l-1}(\omega(z)) \times H^{l-1 / 2}(\partial \omega(z))\right\| .
$$

B случае $l=0$ оченка сохраняется, например, при замене мажоранты на $c\left\|(\mathscr{F}, \mathscr{G}) ; L_{2}(\omega(z)) \times L_{2}(\partial \omega(z))\right\|$. 
2. Задачи на сечении при $j=-2,-1$. В случае $j=-2$ правые части соотношений $(2.10),(2.11)$ равны нулю, т.е. $\mathscr{U}^{(-2)}$ - линейная комбинация векторов (2.14). Обратившись еше раз к формуле (1.15), положим

$$
\mathscr{U}^{(-2)}(z)=e^{1} w_{1}(z)+e^{2} w_{2}(z) .
$$

Рассмотрим теперь задачу $(2.10),(2.11)$ при $j=-1$, т.е.

$$
\begin{aligned}
-\mathbb{D}_{\eta} A \mathbb{D}_{\eta}^{t} \mathscr{U}^{(-1)} & =\mathbb{D}_{\eta} A \mathbb{D}_{z}^{t} \mathscr{U}^{(-2)} \text { в } \omega(z), \\
\mathbb{D}_{\nu} A \mathbb{D}_{\eta}^{t} \mathscr{U}^{(-1)} & =-\mathbb{D}_{\nu} A \mathbb{D}_{z}^{t} \mathscr{U}^{(-2)} \text { на } \partial \omega(z) .
\end{aligned}
$$

При вычислении правых частей в (2.7) учтены равенства (2.5) и тот факт, что функция $(2.16)$ не зависит от $\eta$.

Лемма 2.2. Справедливы соотношения

$$
\mathbb{D}_{z}^{t} e^{i}=\mathbb{D}_{\eta}^{t} e^{3} \eta_{i} \partial_{z}, \quad i=1,2
$$

ДоКАЗАТЕЛЬСТВо достигается непосредственными вычислениями при учете вытекающих из (1.5) и (2.1) формул

$$
\begin{aligned}
\mathbb{D}_{z} & =\left(\begin{array}{cccccc}
0 & 0 & 0 & 0 & \alpha \partial_{z} & 0 \\
0 & 0 & 0 & \alpha \partial_{z} & 0 & 0 \\
0 & 0 & \partial_{z} & 0 & 0 & 0
\end{array}\right), \quad \partial_{z}=\frac{\partial}{\partial z}, \quad \alpha=\frac{1}{\sqrt{2}} \\
\mathbb{D}_{\eta} & =\left(\begin{array}{cccccc}
\partial_{1} & 0 & 0 & 0 & 0 & \alpha \partial_{2} \\
0 & \partial_{2} & 0 & 0 & 0 & \alpha \partial_{1} \\
0 & 0 & 0 & \alpha \partial_{2} & \alpha \partial_{1} & 0
\end{array}\right), \quad \partial_{i}=\frac{\partial}{\partial \eta_{i}}
\end{aligned}
$$

При помощи $(2.18)$ находим частное решение $-\left(\eta_{1} \partial_{z} w_{1}(z)+\eta_{2} \partial_{z} w_{2}(z)\right) e^{3}$ задачи (2.17). Теперь положим

$$
\mathscr{U}^{(-1)}(\eta, z)=w_{3}(z) e^{3}+w_{4}(z) \Phi^{4}(\eta)-e^{3} \sum_{i=1}^{2} \eta_{i} \partial_{z} w_{i}(z) .
$$

ЗАмечАниЕ 2.3. В определении решения (2.20) имеется произвол (предложение 2.1). При условии соблюдения (2.16) он не сказьвается на процедуре в целом. Решение (2.20) было фиксировано так, чтобы сумма $h^{-2} \mathscr{U}^{(-2)}+h^{-1} \mathscr{U}^{(-1)}$ описывала жесткие смещения стержня посредством наиболее простых формул для $w_{1}, \ldots, w_{4}$. Именно, согласно (2.16) и (2.20) случаю $w_{m}=c_{m}, m=1,2,3$, и $w_{4}=0$ отвечает жесткое поступательное смещение, а случаю $w_{i}(z)=C_{i} z$, $i=1,2, w_{3}=0$ и $w_{4}=C_{3}$ - врашательное. При этом член $\mathscr{U}^{(-1)}$ в (2.9) зависит от выбора координат (нужно “отметить" ось стержня), но член $\mathscr{U}^{(-2)}$ при параллельном переносе декартовой системы остается неизменным - он и является ключевым фрагментом в анзаце (2.9) или (1.15). 
3. Задача на сечении при $j=0$. Имеем

$$
\begin{aligned}
L^{0} \mathscr{U}^{(0)}= & \mathbb{D}_{\eta} A \mathbb{D}_{z}^{t} \mathscr{U}^{(-1)}+\mathbb{D}_{z} A\left\{\mathbb{D}_{\eta}^{t} \mathscr{U}^{(-1)}+\mathbb{D}_{z}^{t} \mathscr{U}^{(-2)}\right\} \text { в } \omega(z), \\
B^{0} \mathscr{U}^{(0)}= & -\mathbb{D}_{\nu} A \mathbb{D}_{z}^{t} \mathscr{U}^{(-1)} \\
& -\nu_{0} \mathbb{D}_{1} A\left\{\mathbb{D}_{\eta}^{t} \mathscr{U}^{(-1)}+\mathbb{D}_{z}^{t} \mathscr{U}^{(-2)}\right\} \text { на } \partial \omega(z) .
\end{aligned}
$$

В силу $(2.16),(2.20)$ и (2.18) выражение в фигурных скобках равно нулю. Кроме того, при любом столбце $\mathscr{W} \in H^{1}(\omega(z))^{6}$

$$
\begin{aligned}
\left(\Phi^{q}, \mathbb{D}_{\eta} \mathscr{W}\right)_{\omega(z)} & -\left(\Phi^{q}, \mathbb{D}_{\nu} \mathscr{W}\right)_{\partial \omega(z)}=\left(\mathbb{D}_{\eta}^{t} \Phi^{q}, \mathscr{W}\right)_{\omega(z)} \\
& =(0, \mathscr{W})_{\omega(z)}=0, \quad q=1, \ldots, 4 .
\end{aligned}
$$

Следовательно, правые части (2.21) удовлетворяют (2.13), а сама задача (2.21) разрешима.

Лемма 2.4. Справедлива формула

$$
\mathbb{D}_{z}^{t} \mathscr{U}^{(-1)}(\eta, z)=\mathscr{Y}(\eta) \mathscr{D}\left(\partial_{z}\right) w(z),
$$

в которой $w=\left(w_{1}, \ldots, w_{4}\right)^{t}, \mathscr{D}\left(\partial_{z}\right)=\operatorname{diag}\left\{\alpha \partial_{z}^{2}, \alpha \partial_{z}^{2}, \partial_{z}, \partial_{z}\right\} u$

$$
\mathscr{Y}(\eta)^{t}=\left(\begin{array}{cccccc}
0 & 0 & -\alpha^{-1} \eta_{1} & 0 & 0 & 0 \\
0 & 0 & -\alpha^{-1} \eta_{2} & 0 & 0 & 0 \\
0 & 0 & 1 & 0 & 0 & 0 \\
0 & 0 & 0 & \alpha^{2} \eta_{1} & -\alpha^{2} \eta_{2} & 0
\end{array}\right) .
$$

ДокАЗАТЕЛЬСТво получается непосредственным применением формул (2.19) и $(2.20)$.

Итак,

$$
\mathscr{U}^{(0)}(\eta, z)=\mathscr{V}(\eta, z) \mathscr{D}\left(\partial_{z}\right) w(z),
$$

где $\mathscr{V}-(3 \times 4)$-матрица, удовлетворяющая уравнениям

$$
\begin{aligned}
-\mathbb{D}_{\eta} A \mathbb{D}_{\eta}^{t} \mathscr{V} & =\mathbb{D}_{\eta} A \mathscr{Y} \text { в } \quad \omega(z), \\
\mathbb{D}_{\nu} A \mathbb{D}_{\eta}^{t} \mathscr{V} & =-\mathbb{D}_{\nu} A \mathscr{Y} \quad \text { на } \quad \partial \omega(z) .
\end{aligned}
$$

Иными словами, каждый столбец матрицы $\mathscr{V}$ является решением задачи $(2.12)$ с правыми частями специального вида, которые подчинены условиям разрешимости (2.13) в силу (2.22). Эти столбцы нормируются соотношениями (2.15).

4. Задача на сечении при $j=1$. Найдем условия разрешимости задачи

$$
\begin{aligned}
L^{0} \mathscr{U}^{(1)}= & \mathbb{D}_{\eta} A \mathbb{D}_{z}^{t} \mathscr{U}^{(0)}+\mathbb{D}_{z} A\left\{\mathbb{D}_{\eta} \mathscr{U}^{(0)}+\mathbb{D}_{z}^{t} \mathscr{U}^{(-1)}\right\}+f^{0} \quad \text { в } \omega(z), \\
B^{0} \mathscr{U}^{(1)}= & -\mathbb{D}_{\nu} A \mathbb{D}_{z}^{t} \mathscr{U}^{(0)} \\
& -\nu_{0} \mathbb{D}_{1} A\left\{\mathbb{D}_{\eta} \mathscr{U}^{(0)}+\mathbb{D}_{z}^{t} \mathscr{U}^{(-1)}\right\}+g^{0} \text { на } \partial \omega(z) .
\end{aligned}
$$


Лемма 2.5 (см. [30, 2]). Пусть $Y$ - гладкая функиия на $\bar{\Omega}_{1}$ (см. (1.1)). Тогда при $z \in[-1,1]$

$$
\frac{d}{d z} \int_{\omega(z)} Y(\eta, z) d \eta=\int_{\omega(z)} \frac{\partial Y}{\partial z}(\eta, z) d \eta-\int_{\partial \omega(z)} Y(\eta, z) \nu_{0}(\eta, z) d s_{\eta}
$$

ДоКАЗАТЕЛЬСТВО. При помощи разбиения единицы ситуацию удается упростить дополнительным условием малости носителя подынтегрального выражения. Тогда можно считать, что вблизи supp $Y$ область $\omega(z)$ задана неравенством $\eta_{2}<$ $F\left(\eta_{1}, z\right)$. Формула $(2.2)$ конкретизируется так:

$$
\nu_{1}=-\mathscr{N}^{-1 / 2} \frac{\partial F}{\partial \eta_{1}}, \quad \nu_{2}=-\mathscr{N}^{1 / 2}, \quad \nu_{0}=-\mathscr{N}^{-1 / 2} \frac{\partial F}{\partial z}, \quad \mathscr{N}=1+\left|\frac{\partial F}{\partial \eta_{1}}\right|^{2} .
$$

Кроме того, при некоторых $a_{ \pm}$и $b$

$$
\begin{aligned}
\frac{d}{d z} \int_{\omega(z)} Y(\eta, z) d \eta & =\frac{d}{d z} \int_{a_{-}}^{a_{+}} \int_{b}^{F\left(\eta_{1}, z\right)} Y(\eta, z) d \eta_{1} d \eta_{2} \\
& =\int_{\omega(z)} \frac{\partial Y}{\partial z}(\eta, z) d \eta+\int_{a_{-}}^{a_{+}} Y(\eta, z) \frac{\partial F}{\partial z}\left(\eta_{1}, z\right) d \eta_{1} .
\end{aligned}
$$

Осталось заметить, что на рассматриваемом участке гранищы $d s_{\eta}=\mathscr{N}^{1 / 2} d \eta_{1}$ (элемент длины дуги).

Ввиду (2.22) первые слагаемые в правых частях (2.27) не влияют на условия разрешимости. Благодаря формулам $(2.28),(2.18)$ и предположению $(2.7)$ получаем, что при $i=1,2$

$$
\begin{aligned}
\left(e^{i}, f^{0}+\right. & \left.\partial_{z} \mathbb{D}_{1} A\left\{\mathbb{D}_{\eta}^{t} \mathscr{U}^{(0)}+\mathbb{D}_{z}^{t} \mathscr{U}^{(-1)}\right\}\right)_{\omega(z)} \\
& +\left(e^{i}, g^{0}-\nu_{0} \mathbb{D}_{1} A\left\{\mathbb{D}_{\eta} \mathscr{U}^{(0)}+\mathbb{D}_{z}^{t} \mathscr{U}^{(-1)}\right\}\right)_{\partial \omega(z)} \\
= & \partial_{z}\left(\mathbb{D}_{1}^{t} e^{i}, A\left\{\mathbb{D}_{\eta}^{t} \mathscr{U}^{(0)}+\mathbb{D}_{z}^{t} \mathscr{U}^{(-1)}\right\}\right)_{\omega(z)} \\
= & \partial_{z}\left(\mathbb{D}_{\eta}^{t} e^{3} \eta_{i}, A\left\{\mathbb{D}_{\eta}^{t} \mathscr{U}^{(0)}+\mathbb{D}_{z}^{t} \mathscr{U}^{(-1)}\right\}\right)_{\omega(z)} \\
= & -\partial_{z}\left(e^{3} \eta_{i}, \mathbb{D}_{\eta} A\left\{\mathbb{D}_{\eta}^{t} \mathscr{U}^{(0)}+\mathbb{D}_{z}^{t} \mathscr{U}^{(-1)}\right\}\right)_{\omega(z)} \\
& +\partial_{z}\left(e^{3} \eta_{i}, \mathbb{D}_{\nu} A\left\{\mathbb{D}_{\eta}^{t} \mathscr{U}^{(0)}+\mathbb{D}_{z}^{t} \mathscr{U}^{(-1)}\right\}\right)_{\partial \omega(z)} .
\end{aligned}
$$

Вспоминая, что в равенствах (2.21) фигурные скобки равны нулю, видим, что правая часть (2.29) исчезает, т.е. для задачи (2.27) выполняются условия разрешимости $(2.13)$ с $q=1,2$.

Преобразуем те же условия при $q=3,4$ :

$$
\begin{aligned}
0= & \left(\Phi^{q}, \mathbb{D}_{z} A\left\{\mathbb{D}_{\eta}^{t} \mathscr{U}^{(0)}+\mathbb{D}_{z}^{t} \mathscr{U}^{(-1)}\right\}\right)_{\omega(z)} \\
& -\left(\Phi^{q}, \nu_{0} \mathbb{D}_{1} A\left\{\mathbb{D}_{\eta}^{t} \mathscr{U}^{(0)}+\mathbb{D}_{z}^{t} \mathscr{U}^{(-1)}\right\}\right)_{\partial \omega(z)}+\mathscr{F}^{q},
\end{aligned}
$$


здесь

$$
\mathscr{F}^{q}=\left(\Phi^{q}, f^{0}\right)_{\omega(z)}+\left(\Phi^{q}, g^{0}\right) \partial \omega(z) \cdot
$$

В силу $(2.28),(2.23)$ и (2.25) равенства (2.30) эквивалентны следующим:

$$
-\partial_{z}\left(\mathbb{D}_{1}^{t} \Phi^{q}, A\left\{\mathbb{D}_{\eta}^{t} \mathcal{V}+\mathscr{Y}\right\}\right)_{\omega(z)} \mathscr{D}\left(\partial_{z}\right) w(z)=\mathscr{F}^{q}(z), \quad z \in(-1,1), \quad q=3,4
$$

Итак, найдены два уравнения для определения неизвестного вектора $w=$ $\left(w_{1}, \ldots, w_{4}\right)^{t}$. Далее важным окажется то, что $\mathbb{D}_{1}^{t} \Phi^{3}$ и $\mathbb{D}_{1}^{t} \Phi^{4}$ совпадают с третьим и четвертым столбцами матрицы و из (2.24).

5. Два условия разрешимости задачи на сечении при $j=2$. Если предположить, что в (2.6) $\tilde{f}=0, \tilde{g}=0$, то для определения $\mathscr{U}^{(2)}$ появится краевая задача:

$$
\begin{aligned}
& L^{0} \mathscr{U}^{(2)}=\mathbb{D}_{\eta} A \mathbb{D}_{z}^{t} \mathcal{U}^{(1)}+\mathbb{D}_{z} A\left\{\mathbb{D}_{\eta}^{t} \mathscr{U}^{(1)}+\mathbb{D}_{z}^{t} \mathscr{U}^{(0)}\right\}+\bar{f}^{0} \quad \text { в } \omega(z), \\
& B^{0} \mathscr{U}^{(2)}=-\mathbb{D}_{\nu} A \mathbb{D}_{z}^{t} \mathscr{U}^{(1)}-\nu_{0} \mathbb{D}_{1} A\left\{\mathbb{D}_{\eta}^{t} \mathscr{U}^{(1)}+\mathbb{D}_{z}^{t} \mathscr{U}^{(0)}\right\} \text { на } \partial \omega(z) .
\end{aligned}
$$

Рассмотрим первые два $(q=1,2)$ условия разрешимости (2.13). Как обычно, благодаря (2.22) на первые слагаемые справа в (2.33) можно не обращать внимания. Применяя леммы 2.5, 2.2 и интегрируя по частям, находим

$$
\begin{aligned}
& \left(e^{i}, \mathbb{D}_{z} A\left\{\mathbb{D}_{\eta}^{t} \mathscr{U}^{(1)}+\mathbb{D}_{z}^{t} \mathscr{U}^{(0)}\right\}+\bar{f}^{0}\right)_{\omega(z)}-\left(e^{i}, \nu_{0} \mathbb{D}_{1} A\left\{\mathbb{D}_{\eta}^{t} \mathscr{U}^{(1)}+\mathbb{D}_{z}^{t} \mathscr{U}^{(0)}\right\}\right)_{\partial \omega(z)} \\
& =|\omega(z)| \bar{f}^{0}+\partial_{z}\left(e^{i}, \mathbb{D}_{1} A\left\{\mathbb{D}_{\eta}^{t} \mathscr{U}^{(1)}+\mathbb{D}_{z}^{t} \mathscr{U}^{(0)}\right\}\right)_{\omega(z)}, \\
& \left(\mathbb{D}_{1}^{t} e^{i}, A\left\{\mathbb{D}_{\eta}^{t} \mathcal{U}^{(1)}+\mathbb{D}_{z}^{t} \mathscr{U}^{(0)}\right\}\right)_{\omega(z)}=\left(\mathbb{D}_{\eta}^{t} e^{3} \eta_{i}, A\left\{\mathbb{D}_{\eta}^{t} \mathscr{U}^{(1)}+\mathbb{D}_{z}^{t} \mathscr{U}^{(0)}\right\}\right)_{\omega(z)} \\
& =-\left(e^{i} \eta_{i}, \mathbb{D}_{\eta} A\left\{\mathbb{D}_{\eta}^{t} \mathscr{U}^{(1)}+\mathbb{D}_{z}^{t} \boldsymbol{U}^{(0)}\right\}\right)_{\omega(z)} \\
& +\left(\eta_{i} e^{3}, \mathbb{D}_{\nu} A\left\{\mathbb{D}_{\eta}^{t} \mathscr{U}^{(1)}+\mathbb{D}_{z}^{t} \mathscr{U}^{(0)}\right\}\right)_{\partial \omega(z)} \equiv \Sigma_{i}
\end{aligned}
$$

Здесь через $|\omega(z)|$ обозначена площадь $\operatorname{mes}_{2} \omega(z)$ сечения. Выражения $\Sigma_{i}, i=1,2$, преобразуем при помоши равенств (2.27), а также леммы 2.5 и формул $(2.25),(2.23)$,

$$
\begin{aligned}
\Sigma_{i}= & \left(\eta_{i} e^{3}, \mathbb{D}_{z} A\left\{\mathbb{D}_{\eta}^{t} \mathscr{U}^{(0)}+\mathbb{D}_{z}^{t} \mathscr{U}^{(-1)}\right\}\right)_{\omega(z)}+\left(\eta_{i}, f_{3}^{0}\right)_{\omega(z)} \\
& -\left(\eta_{i} e^{3}, \nu_{0} \mathbb{D}_{1} A\left\{\mathbb{D}_{\eta}^{t} \mathscr{U}^{(0)}+\mathbb{D}_{z}^{t} \mathscr{U}^{(-1)}\right\}\right)_{\partial \omega(z)}+\left(\eta_{i}, g_{3}^{0}\right)_{\partial \omega(z)} \\
= & \left(\eta_{i}, f_{3}^{0}\right)_{\omega(z)}+\left(\eta_{i}, g_{3}^{0}\right)_{\partial \omega(z)}+\partial_{z}\left(\mathbb{D}_{1}^{t} \eta_{i} e^{3}, A\left\{\mathbb{D}_{\eta}^{t} \mathscr{V}+\mathscr{Y}\right\}\right)_{\omega(z)} \mathscr{D}\left(\partial_{z}\right) w(z) .
\end{aligned}
$$

Итак, два условия разрешимости задачи (2.33) принимают вид обыкновенных дифференциальных уравнений

$$
-\partial_{z}^{2}\left(\eta_{i} \mathbb{D}_{1}^{t} e^{3}, A\left\{\mathbb{D}_{\eta}^{t} \mathscr{V}+\mathscr{Y}\right\}\right)_{\omega(z)} \mathscr{D}\left(\partial_{z}\right) w(z)=\mathscr{F}_{i}(z), \quad z \in(-1,1),
$$

где $i=1,2$ и

$$
\mathscr{F}_{i}(z)=|\omega(z)| \bar{f}_{i}^{0}(z)+\partial_{z}\left\{\left(\eta_{i}, f_{3}^{0}(\eta, z)\right)_{\omega(z)}+\left(\eta_{i}, g_{3}^{0}(\eta, z)\right)_{\partial \omega(z)}\right\}
$$


В дальнейших асимптотических конструкциях вектор $\mathscr{U}^{(2)}$ участия не принимает (см. § 4), однако введение условий (2.34) не обязательно связывать с разрешимостью задачи (2.33). Сумма четырех слагаемых справа в $(2.9)\left(\right.$ без $\left.h^{2} \mathscr{U}^{(2)}+\cdots\right)$ оставляет невязки в системе (1.6) и краевом условии (1.7), которые обладают той же структурой, что и исходные правые части (2.6), с дополнительным множителем $h$. Иньми словами, в принципе процедуру построения формальной асимптотики можно продолжить, для чего нужно обеспечить аналогичное (2.7) условие ортогональности (напомним, что $\mathscr{U}^{(1)}$ определяется только при учете (2.7)). Роль таких условий и играют уравнения (2.34). Подчеркнем, что в п. $3 \S 4$ при обосновании асимптотики условия ортогональности будут востребованы еше раз.

6. Результирующая задача. Согласно $(2.24)$ и $(2.19)$ столбец $-\alpha^{-1} \eta_{i} \mathbb{D}_{1}^{t} e^{3}$ при $i=1,2$ фигурирует и в матрище $\mathscr{Y}(\eta)$, и в скалярном произведении из $(2.34)$. $\mathrm{B}$ силу аналогичного наблюдения относительно столбцов $\mathbb{D}_{1}^{t} \Phi^{q}$ в $(2.32)$ переписываем дифференциальные уравнения (2.32) и (2.34) одной строкой:

$$
\mathscr{D}\left(-\partial_{z}\right) \mathscr{M}(z) \mathscr{D}\left(\partial_{z}\right) w(z)=\mathscr{F}(z), \quad z \in(-1,1),
$$

где $\mathscr{F}=\left(\mathscr{F}_{1}, \ldots, \mathscr{F}_{4}\right)^{t}$ и $\mathscr{M}-(4 \times 4)$-матрица-функция,

$$
\mathscr{M}(z)=\int_{\omega(z)} \mathscr{Y}(\eta)^{t} A(\eta, z)\left(\mathbb{D}_{\eta}^{t} \mathscr{V}(\eta, z)+\mathscr{Y}(\eta)\right) d \eta
$$

В соответствии с (1.8) дополним систему (2.36) условиями Дирихле на концах интервала $(-1,1)$ :

$$
w_{q}( \pm 1)=0, \quad q=1, \ldots, 4, \quad \partial_{z} w_{i}( \pm 1)=0, \quad i=1,2 .
$$

ПРЕДЛОЖЕНИЕ 2.6. При любом $z \in(-1,1)$ матрица (2.37) симметрическая и положительно определенная.

ДоКАЗАТЕЛЬСТВО. Пользуясь уравнениями (2.26) и интегрируя по частям, находим, что

$$
\begin{aligned}
\int_{\omega(z)}\left(\mathbb{D}_{\eta}^{t} \mathscr{V}\right. & +\mathscr{Y})^{t} A\left(\mathbb{D}_{\eta}^{t} \mathscr{V}+\mathscr{Y}\right) d \eta=\mathscr{M}+\int_{\omega(z)}\left(\mathbb{D}_{\eta}^{t} \mathscr{V}\right)^{t} A\left(\mathbb{D}_{\eta}^{t} \mathscr{V}+\mathscr{Y}\right) d \eta \\
= & \mathscr{M}-\int_{\omega(z)} \mathscr{V}^{t} \mathbb{D}_{\eta} A\left(\mathbb{D}_{\eta}^{t \mathscr{V}}+\mathscr{Y}\right) d \eta \\
& +\int_{\partial \omega(z)} \mathscr{V}^{t} \mathbb{D}_{\nu} A\left(\mathbb{D}_{\eta}^{t} \mathscr{V}+\mathscr{Y}\right) d s_{\eta}=\mathscr{M} .
\end{aligned}
$$

Таким образом, $\mathscr{M}$ - матрица Грама, построенная по столбцам $(6 \times 4)$-матрицы $\mathbb{D}_{\eta}^{t} \mathscr{V}+\mathscr{Y}$ при помощи скалярного произведения $\langle u, v\rangle=(u, A v)_{\omega(z)}$ в $L_{2}(\omega(z))^{6}$ (напоминаем про симметричность и положительную определенность $A$ ). Осталось убедиться в том, что названные столбцы линейно независимы. Пусть $a \in \mathbb{R}^{4}$ и

$$
\mathbb{D}_{\eta}^{t} \mathscr{V} a+\mathscr{Y} a=0
$$


В силу (2.19) и (2.24) третья строка системы (2.40) читается так:

$$
0+(\mathscr{Y} a)_{3}=-\alpha^{-1} \eta_{1} a_{1}-\alpha^{-1} \eta_{2} a_{2}+a_{3}=0 .
$$

Значит, $a_{1}=a_{2}=a_{3}=0$. Кроме того, четвертая и пятая строки дают соотношения

$$
\alpha \partial_{2}(\mathscr{V} a)_{3}+\alpha^{2} \eta_{1} a_{4}=0, \quad \alpha \partial_{1}(\mathscr{V} a)_{3}-\alpha^{2} \eta_{2} a_{4}=0,
$$

откуда дифференцированием выводим, что $2 \alpha^{2} a_{4}=0$. Искомая тривиальность столбца $а$ установлена.

Очередное утверждение стало очевидным.

Teоpema 2.7. Если $\mathscr{F}_{i} \in H_{2}^{l}(-1,1), \quad \mathscr{F}_{2+i} \in H_{2}^{l-1}(-1,1)$ при некотором $l \in \mathbb{N}$ и $i=1,2$, то существует единственное решение задачи (2.36), (2.38) и верна оченка

$$
\left\|w ; H_{2}^{l+3}(-1,1)^{2} \times H_{2}^{l+2}(-1,1)^{2}\right\| \leqslant c\left\|\mathscr{F} ; H_{2}^{l-1}(-1,1)^{2} \times H_{2}^{l}(-1,1)^{2}\right\| .
$$

ЗАмечание 2.8. Оператор $\mathbf{M}\left(z, \partial_{z}\right)$ системы (2.36) наследует полиномиальное свойство [35] от оператора $L$ из упругой задачи (1.6)-(1.8). Действительно, в силу предложения 2.6 соответствуюшая квадратичная форма $\mathbf{E}(w)$ удовлетворяет неравенству

$$
\mathbf{E}(w) \equiv \int_{-1}^{1}\left(\mathscr{D}\left(\partial_{z}\right) w(z)\right)^{t} \mathscr{M}(z) \mathscr{D}\left(\partial_{z}\right) w(z) d z \geqslant c\left\|\mathscr{D}\left(\partial_{z}\right) w ; L_{2}(-1,1)\right\|^{2}
$$

и поэтому вырождается только на полиномах из линеала

$$
\left\{p=\left(p_{1}, \ldots, p_{4}\right)^{t}: p_{i}(z)=a_{i}+b_{i} z, p_{i+2}=a_{i+2}, a_{i}, a_{i+2}, b_{i} \in \mathbb{R}, i=1,2\right\} .
$$

7. Обсуждение. Как уже упоминалось, представления (2.6) и (1.15) (или (2.9) при учете (2.16) и (2.20)) согласованы. Укажем физическую интерпретацию появившихся математических объектов (всюду имеются в виду главные асимптотические члены механических величин). Усредненные по сечению стержня прогибы в направлении $O y_{i}$ равны $h^{-2} w_{i}(z), i=1,2$, a $h^{-1} w_{3}(z)$ - его смещение вдоль оси $O z$ и $h^{-1} \alpha w_{4}(z)$ - угол закручивания вокруг этой оси. В соответствии с (2.6) и $(2.31),(2.35) h \mathscr{F}_{3}(z)$ и $h^{2} \alpha^{-1} \mathscr{F}_{4}(z)$ - продольная сила и крутящий момент, приложенные к стержню. Кроме того, выражения из фигурных скобок в (2.35) пропорциональны изгибаюшим моментам, а $h^{2}|w(z)| \bar{f}_{i}^{0}(z)$ - перерезывающие силы; здесь $i=1,2$ и $|w(z)|-$ площадь области $w(z)$. Замена в функционале потенциальной энергии

$$
P(u, f, g)=2^{-1} E\left(u, u ; \Omega_{h}\right)-\left[(f, u)_{\Omega_{h}}+(g, u)_{\Gamma_{h}}\right]
$$

векторов $u, f$ и $g$ главными членами $h^{-2} \mathscr{U}^{-2}+h^{-1} \mathscr{U}^{-1}+h^{0} \mathscr{U}^{0}, h^{-1} f^{0}+h^{0} \bar{f}^{0}$ и $h^{0} g^{0}$ их асимптотик и последуюший переход к $h=0$ приводят к выражению

$$
\mathbf{P}(w, \mathscr{F})=2^{-1} \mathbf{E}(w)-(\mathscr{F}, w)_{(-1,1)},
$$


являющемуся потенциальной энергией деформации для задачи (2.36), (2.38). Это наблюдение будет обосновано в п. $4 \S 4$. Его вывод сушественно опирается на формулы $(2.16),(2.6),(2.7)$ и $(2.37)$ - без условия ортогональности (2.7) вычитаемое в (2.43) не имеет конечного предела при $h \rightarrow+0$.

При вычислении асимптотик пространственных полей деформаций и напряжений необходимо учитывать три слагаемых $(2.16),(2.20)$ и $(2.25)$ в представлении (2.9) поля смешений. K этому вопросу мы вернемся в п. $2 \S 3$ и п. $4 \S 4$.

Если $\Omega_{h}$ - искривленный стержень с постоянным поперечным сечением (в (1.1) $\omega(z)$ зависит от $z$ специальным образом), то оправдан переход к натуральным криволинейным координатам, связанным с осью стержня. При этом поперечные смещения получаются в главном простым ортогональным преобразованием $\left(w_{1}, w_{2}\right)$, а продольное смещение и закручивание требуют пересчета, базирующегося на полном анзаце (1.15), так как при поворотах осей на углы $O(h)$ старший член асимптотики $h^{-2}\left(w_{1} e^{1}+w_{2} e^{2}\right)$ оказывает влияние на коэффициент при $h^{-1}$ (ср. с замечанием 2.3).

\section{§ 3. Весовое неравенство Корна}

1. Дополнительные геометрические предположения. Для обоснования формальной асимптотики, построенной в $\S 2$, необходима правильная запись неравенства Корна в тонкой искривленной области. Асимптотическая точность такого неравенства достигается введением в $L_{2}$-нормы смешений и их производных добавочных весовых множителей - степеней параметра $h$ и расстояний до торцов стержня. Для тонких цилиндрических областей подобные неравенства получены в [36], [33] (изотропное распределение весовых множителей) и в [32], [34] (анизотропное распределение, выделяющее направление вдоль оси стержня). В этом параграфе весовое неравенство Корна обобщается на случай криволинейного стержня при дополнительных предположениях о его геометрии. Сформулируем их.

Разобьем область $\Omega_{h}$ на ячейки $S_{m}=\left\{x \in \Omega_{h}: h m<z<h(m+1)\right\} ;$ здесь $m$ - целое число. Пусть еще $\xi^{ \pm}$- некоторая система декартовых координат, центр которой помещен на торец $\omega_{h}( \pm 1)$ стержня $\Omega_{h}$. Введем круговые цилиндры $Q_{h}^{ \pm}=$ $\left\{x:\left|\xi_{1}^{ \pm}\right|^{2}+\left|\xi_{2}^{ \pm}\right|^{2}<4 h^{2} R_{ \pm}^{2},-C_{ \pm} h<\xi_{3}^{ \pm}<H_{ \pm}\right\} ;$при этом

$$
0<R_{ \pm} \leqslant \frac{1}{2}, \quad, C_{ \pm} \geqslant 1, \quad 0<H_{ \pm}<2
$$

Предположим, что системы координат $\xi^{ \pm}$и не зависяшие от $h$ числа $R_{ \pm}, C_{ \pm}, H_{ \pm}$ удается подобрать так, чтобы были соблюдены условия:

i) основные части цилиндров $Q_{h}^{ \pm}$лежат внутри $\Omega_{h}$, т.е. $\left\{Q_{h}^{ \pm}: \pm z<1\right\} \subset \Omega_{h}$;

ii) каждая из ячеек $S_{m}$, составляющих $\Omega_{h}$, пронизана хотя бы одним из стержней $Q_{h}^{ \pm}$, т.е. $S_{m} \cap Q_{h}^{ \pm}=\left\{x \in Q_{h}^{ \pm}: h m<z<h(m+1)\right\}$.

Из іi) вытекает, в частности, что $H_{+}+H_{-} \geqslant 2$. Типичная картина изображена на рис. 1. 


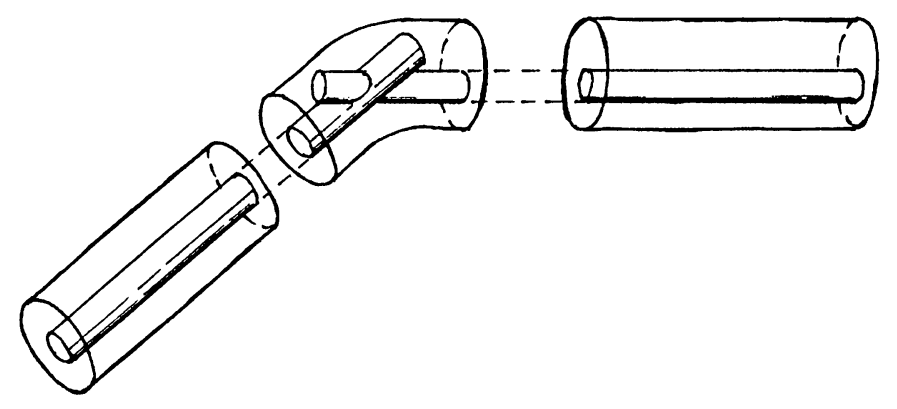

Рис. 1

Вектор-функция $u \in \stackrel{\circ}{H^{1}}\left(\Omega_{h}\right)^{3}$ продолжается нулем на полупространства $\{x$ : $\pm z>1\}$ с сохранением класса. Поэтому ее можно считать заданной на $Q_{h}^{ \pm}$, причем $u=0$ на торце $q_{h}^{ \pm}=\left\{x \in \bar{Q}_{h}^{ \pm}: \xi_{3}^{ \pm}=-C_{ \pm} h\right\}$ (поскольку в силу (3.1) $q_{h}^{ \pm}$лежит вне $\Omega_{h}$ ). Кроме того, если число $1 / h$ не является целым, то крайние ячейки, ограниченные торцами $\omega_{h}( \pm 1)$, имеют меньшую протяженность в направлении $z$, чем средние. Принимая во внимание продолжение поля $u$ нулем, крайние ячейки можно удлинить так, чтобы любая из ячеек $S_{m}$ содержала шар $B_{m}$ с радиусом $h R_{ \pm}$ и центром на пересечении оси цилиндра $Q_{h}^{ \pm}$с плоскостью $\{x: z=h(m+1 / 2)\}$ (знак " \pm " выбираем в соответствии с условием ii) и учитываем первое из неравенств (3.1)). Заметим, что соотношения (3.1) не являются ограничительными всегда можно достичь их допустимой вариацией размеров цилиндров.

Последнее из требований заключается в следующем:

iii) справедливо неравенство

$$
\||u|\|_{S_{m}}^{2} \leqslant c\left(E\left(u, u ; S_{m}\right)+\|\| u\|\|_{B_{m}}^{2}\right),
$$

в котором \|\|$u\|\|_{\Xi}^{2}=\left\|\nabla u ; L_{2}(\Xi)\right\|^{2}+h^{-2}\left\|u ; L_{2}(\Xi)\right\|^{2}$, а постоянная $c$ не зависит от $h, m$ и вектор-функции $u \in H^{1}\left(S_{m}\right)^{3}$.

Это требование также можно заменить геометрическим условием: ячейка $S_{m}$ звездна относительно иара $B_{m}$. В самом деле, согласно теореме 1 и замечанию 3 из [31] названное условие обеспечивает оценку

$$
\left\|\nabla_{x} u ; L_{2}\left(S_{m}\right)\right\|^{2} \leqslant c_{1} \varkappa_{m}^{3}\left\{E\left(u, u ; S_{m}\right)+\left\|\nabla_{x} u ; L_{2}\left(B_{m}\right)\right\|^{2}\right\},
$$

в которой $c_{1}$ - абсолютная постоянная, $\varkappa_{m}=R_{m} / R_{ \pm}$, а $R_{m}$ выбрано так, что $S_{m} \subset\left\{x:\left|x-x^{m}\right|<h R_{m}\right\}$, где $x^{m}$ - центр шара $B_{m}$. Ясно, что дробь $\varkappa_{m}$ ограничена постоянной, не зависящей от $m$ и $h$. Таким образом, вместе с легко проверяемым неравенством типа Пуанкаре-Фридрихса

$$
\left\|u ; L_{2}\left(S_{m}\right)\right\|^{2} \leqslant c_{2} \varkappa_{m}^{3}\left\{\left\|u ; L_{2}\left(B_{m}\right)\right\|^{2}+h^{2} R_{m}^{2} \varkappa_{m}^{3}\left\|\nabla_{x} u ; L_{2}\left(S_{m}\right)\right\|^{2}\right\}
$$

оценка (3.2) приводит к нужному неравенству из іiі). 
Требование звездности более сильное, чем само условие іiі). Так, итерированным применением оценок (3.2) и (3.3) можно проверить iii) для стержней с продольньми полостями, выемками и пр. Вообще же, обоснование асимптотики в $₫ 4$ опирается только на окончательный вариант весового неравенства Корна, сформулированный в теореме 3.1 , а геометрические требования i) - iii) вызваны техническими моментами в избранном способе доказательства этой теоремы.

2. Неравенство Корна и его асимптотическая точность. Весовая функция $\rho(z)=h+1-|z|$ принимает значения $O(h)$ в непосредственной близости от торцов стержня $\Omega_{h}$, но на удалении от них становится величиной $O(1)$. Положим

$$
\begin{aligned}
\left|u ; \Omega_{h}, \rho\right|^{2}= & \int_{\Omega_{h}}\left[\left|\frac{\partial u_{i}}{\partial y_{i}}\right|^{2}+\left|\frac{\partial u_{3}}{\partial z}\right|^{2}+\frac{h^{2}}{\rho^{2}}\left\{\left|\frac{\partial u_{1}}{\partial y_{2}}\right|^{2}+\left|\frac{\partial u_{2}}{\partial y_{1}}\right|^{2}\right\}\right. \\
& \left.+\frac{h^{2}}{\rho^{2}}\left\{\left|\frac{\partial u_{i}}{\partial z}\right|^{2}+\left|\frac{\partial u_{3}}{\partial y_{i}}\right|^{2}\right\}+\frac{1}{\rho^{2}}\left\{\left|u_{3}\right|^{2}+\frac{h^{2}}{\rho^{2}}\left|u_{i}\right|^{2}\right\}\right] d y d z
\end{aligned}
$$

Здесь, как и всюду в параграфе, суммирование по $i=1,2$ подразумевается, но не указывается явно.

В этом пункте норму (3.4) обозначаем $|u|$. Так как $\rho(z)>0$ при $x \in \bar{\Omega}_{h}$, она эквивалентна обычной соболевской норме, но, разумеется, одна из констант эквивалентности зависит от малого параметра $h$. Доказательство следующего утверждения приводится в конще параграфа.

ТЕОРемА 3.1. Для вектор-функиии $u \in \stackrel{\circ}{H}^{1}\left(\Omega_{h}\right)^{3}$ справедливо неравенство

$$
|u|^{2} \leqslant c E\left(u, u ; \Omega_{h}\right)
$$

где постоянная с не зависит ни от $и$, ни от $h \in(0,1]$.

Убедимся в асимптотической точности неравенства (3.5) (точнее, в правильности выбора весовой нормы (3.4)). С этой целью возьмем произвольную вектор-функцию $w \in C^{\infty}([-1,1])^{4}$, подчиненную $(2.38)$, построим по ней начальные члены суммы из $(2.9)$ и вычислим порядки относительно $h$ левой и правой частей (3.5).

Положим

$$
\mathscr{W}=h^{-2} \mathscr{U}^{(-2)}+h^{-1} \mathscr{U}^{(-1)}+h^{0} \mathscr{U}^{(0)} ;
$$

в силу $(2.1),(2.16),(2.18),(2.20)$ и $(2.23),(2.25)$ имеем

$$
\begin{aligned}
\varepsilon(\mathscr{W})=D\left(\nabla_{x}\right)^{t} \mathscr{W} & =\left(h^{-1} \mathbb{D}_{\eta}+\mathbb{D}_{z}\right)\left(h^{-2} \mathscr{U}^{(-2)}+h^{-1} \mathscr{U}^{(-1)}+h^{0} \mathscr{U}^{(0)}\right) \\
& =h^{-1}\left(\mathscr{Y}+\mathbb{D}_{\eta} \mathscr{V}\right) \mathscr{D}\left(\partial_{z}\right) w(z)+O(1) .
\end{aligned}
$$

Отсюда и из $(1.10),(2.39)$ вытекает соотношение

$$
E\left(\mathscr{W}, \mathscr{W} ; \Omega_{h}\right)=\mathbf{E}(w)+O(1)
$$


где $2^{-1} \mathbf{E}(\cdot)$ - энергетический функционал для задачи (2.36), (2.38), определенньй в (2.42). Мы ограничились в (3.6) тремя членами, поскольку вклады остальных все равно были бы отнесены в остатки $O(1)$ и $O(h)$; именно до суммы $(3.6)$ урезается асимптотическое разложение (2.9) в центральной теореме 4.4 .

Теперь рассмотрим интеграл из (3.4), содержаший восемь слагаемых $I_{1}, \ldots, I_{8}$. Список соответствуюших подынтегральных выражений таков:

$$
\begin{aligned}
\frac{\partial \mathscr{W}_{p}}{\partial y_{p}} & =\frac{1}{h} \frac{\partial}{\partial \eta_{p}} \mathscr{V}_{p} \mathscr{D}\left(\partial_{z}\right) w, \quad \frac{\partial \mathscr{W}_{3}}{\partial z}=\frac{1}{h}\left(\partial_{z} w_{3}-\eta_{i} \partial_{z}^{2} w_{i}\right)+O(1) \\
-\frac{\partial \mathscr{W}_{1}}{\partial y_{2}}, \frac{\partial \mathscr{W}_{2}}{\partial y_{1}} & =\frac{\alpha}{h^{2}} w_{4}+O\left(\frac{1}{h}\right), \quad-\frac{\partial \mathscr{W}_{3}}{\partial y_{p}}, \frac{\partial \mathscr{W}_{p}}{\partial z}=\frac{1}{h^{2}} \partial_{z} w_{p}(z)+O\left(\frac{1}{h}\right) \\
\mathscr{W}_{3} & =h^{-1}\left(w_{3}-\eta_{i} \partial_{z} w_{i}\right)+O(1), \quad \mathscr{W}_{p}=h^{-2} w_{p}+O\left(h^{-1}\right)
\end{aligned}
$$

здесь $p=1,2$. Нетрудно убедиться в следующем: степени $h$ распределены в (3.4) так, что каждый из интегралов $I_{j}$ равен $O(1)$, а значит, $|\mathscr{W}|^{2}=O(1)$. Этот факт, вкупе с формулой (3.7) и теоремой 4.4 , обосновываюшей асимптотику, указывает на асимптотическую точность оценки (3.5). Отметим еще, что ввиду (2.38)

$$
w_{p}(z)=O\left([1-|z|]^{2}\right), \quad w_{2+p}(z)=O([1-|z|]) .
$$

Просматривая список (3.8) еше раз, видим, что весовые множители $\rho^{-2}$ расставлены в (3.4) с соблюдением условия: их влияние на сходимость интеграла компенсируется малостью выражений (3.8) при $z \rightarrow \pm 1$, обеспечиваемой соотношениями (3.9).

3. Весовое неравенство Корна на $Q_{h}^{ \pm}$. Ввиду симметрии имеем дело только с цилиндром $Q_{h}^{-}$, а индексы “土” не указываем в обозначениях. Вспомнив сказанное в п. 1 о продолжении нулем вектор-функции $u \in \stackrel{\circ}{H^{1}}\left(\Omega_{h}\right)^{3}$, считаем, что $u \in \stackrel{\circ}{H^{1}}\left(Q_{h}\right)^{3}$ (т.е. $u$ обрашается в нуль на $q_{h}$ ). Пусть $v_{1}, v_{2}$ и $v_{3}-$ проекции вектоpa $u$ на оси $\xi_{1}, \xi_{2}$ и $\xi_{3}$. Радиус коаксиального с $Q_{h}$ цилиндра $Q_{h}^{\prime}=\left\{\xi \in Q_{h}: \xi_{1}^{2}+\right.$ $\left.\xi_{2}^{2} \leqslant h^{2} R^{2}\right\}$ уменьшим вдвое. Временно положим

$$
\begin{aligned}
|v|^{2}= & \int_{Q_{h}^{\prime}}\left[\left|\frac{\partial v_{i}}{\partial \xi_{i}}\right|^{2}+\left|\frac{\partial v_{3}}{\partial \xi_{3}}\right|^{2}+\frac{h^{2}}{\left(h+\xi_{3}\right)^{2}}\left\{\left|\frac{\partial v_{1}}{\partial \xi_{2}}\right|^{2}+\left|\frac{\partial v_{2}}{\partial \xi_{1}}\right|^{2}\right\}\right. \\
& +\frac{h^{2}}{\left(h+\xi_{3}\right)^{2}}\left\{\left|\frac{\partial v_{i}}{\partial \xi_{3}}\right|^{2}+\left|\frac{\partial v_{3}}{\partial \xi_{i}}\right|^{2}\right\} \\
& \left.+\frac{1}{\left(h+\xi_{3}\right)^{2}}\left\{\left|v_{3}\right|^{2}+\frac{h^{2}}{\left(h+\xi_{3}\right)^{2}}\left|v_{i}\right|^{2}\right\}\right] d \xi,
\end{aligned}
$$

т.е. приспособим весовую норму (3.4) к положению цилиндра $Q_{h}$. Так как число $H$ из (3.1) не зависит от $h$, угол между осями $\xi_{3}$ и $z$ составляет $O(1)$, и, следовательно, используемые декартовы координаты связаны формулой

$$
(y, z+1)=\Theta \xi
$$


где $\Theta$ - унитарная матрища, элементы которой удовлетворяют неравенству

$$
\left|\Theta_{p q}\right|+\left|\Theta_{33}\right|+h^{-1}\left|\Theta_{p 3}\right|+h^{-1}|\Theta|_{3 q} \leqslant C, \quad p, q=1,2,
$$

с постоянной $C$, не зависящей от $h$. Таким образом,

$$
u=\Theta v, \quad \nabla_{\xi}=\Theta \nabla_{x}
$$

Лемма 3.2. Для $u \in \stackrel{\circ}{H^{1}}\left(Q_{h}\right)^{3}$ справедливы неравенства

$$
\begin{gathered}
|v|^{2} \leqslant C E\left(u, u ; Q_{h}\right), \\
\left|u ; Q_{h}^{\prime}, \rho\right|^{2} \leqslant c\left(|v|^{2}+E\left(u, u ; Q_{h}^{\prime}\right)\right),
\end{gathered}
$$

в которых с и $C$ не зависят от $u, h$.

ДокАзАтЕльство. Сначала заметим, что энергетический функционал невосприимчив к ортогональному преобразованию координат. Если множитель перед первой фигурной скобкой в $(3.10)$ заменить на $h^{4}\left(h+\xi_{3}\right)^{-4}$, то неравенство $|v|^{2} \leqslant$ $C E\left(u, u ; Q_{h}\right)$ совпадет с неравенством, доказанным в [32] (см. также [34, предложение 3.1]). Оценка

$$
h^{2} \int_{Q_{h}^{\prime}}\left(h+\xi_{3}\right)^{-2}\left|\nabla_{\xi} v_{i}\right|^{2} d \xi \leqslant C E\left(u, u ; Q_{h}\right)
$$

достаточная теперь для проверки (3.13), по сушеству установлена в [33, лемма 1] при помощи техники, разработанной в [37], [36], [31] (см. [38, лемма 3.1]).

Перейдем к доказательству (3.14). С этой целью сравним выражение (3.10) с выражением (3.4), где интегрирование ведется по $Q_{h}^{\prime}$. Весовые множители $\rho$ и $h+\xi_{3}$ эквивалентны. В силу (3.11) и (3.12)

$$
\left|u_{p}\right| \leqslant c\left(\left|v_{1}\right|+\left|v_{2}\right|+h\left|v_{3}\right|\right), \quad\left|u_{3}\right| \leqslant c\left(\left|v_{3}\right|+h\left|v_{1}\right|+h\left|v_{2}\right|\right) .
$$

Так как $h \leqslant \rho(z) \leqslant 4$, получаем

$$
\begin{aligned}
\left|u_{3}\right|^{2}+h^{2} \rho^{-2}\left|u_{i}\right|^{2} & \leqslant c_{1}\left\{\left|v_{3}\right|^{2}+h^{2}\left|v_{i}\right|^{2}+h^{2} \rho^{-2}\left(\left|v_{i}\right|^{2}+h^{2}\left|v_{3}\right|^{2}\right)\right\} \\
& \leqslant c_{2}\left(\left|v_{3}\right|^{2}+h^{2} \rho^{-2}\left|v_{i}\right|^{2}\right) \leqslant c_{3}\left(\left|v_{3}\right|^{2}+h^{2}\left(h+\xi_{3}\right)^{-2}\left|v_{i}\right|^{2}\right) .
\end{aligned}
$$

Таким образом, фрагменты интегралов из (3.4) и (3.10), содержащие только сами компоненты вектора смещений, нужным неравенством связаны. Для деформаций $\partial u_{i} / \partial y_{i}$ и $\partial u_{3} / \partial z$ требуемые оценки очевидны, поскольку согласно $(1.2)-(1.4)$ и (1.10) квадраты их норм в $L_{2}\left(Q_{h}^{\prime}\right)$ мажорируются величиной $c E\left(u, u ; Q_{h}^{\prime}\right)$. Наконец, правая часть формулы

$$
c \frac{h^{2}}{\rho^{2}}\left\{\left|\frac{\partial u_{1}}{\partial y_{2}}\right|^{2}+\left|\frac{\partial u_{2}}{\partial y_{1}}\right|^{2}+\left|\frac{\partial u_{i}}{\partial z}\right|^{2}+\left|\frac{\partial u_{3}}{\partial y_{i}}\right|^{2}\right\} \leqslant \frac{h^{2}}{\left(h+\xi_{3}\right)^{2}} \sum_{j=1}^{3}\left|\nabla_{\xi} v_{j}\right|^{2},
$$

вытекаюшей из (3.11) и (3.12), не больше подынтегрального выражения в (3.10). Проверка (3.14) закончена. 
4. Доказательство теоремы 3.1. Рассмотрим одну из ячеек $S_{m}$, на которые разбит стержень $\Omega_{h}$, и расположенньй в ней шар $B_{m}$ с радиусом $h R_{ \pm}$и центром $x^{m}=\left(y_{1}^{m}, y_{2}^{m}, h(m+1 / 2)\right)$. По построению этот шар принадлежит также одному из круговых цилиндров $Q_{h}^{\prime \pm}$ (их радиусы $h R_{ \pm}$вдвое меньше радиусов цилиндров $Q_{h}^{ \pm}$, фигурируюших в предположении іi)). Для определенности будем считать, что $B_{m} \subset Q_{h}^{\prime-}$. Тогда

$$
c \rho(x) \leqslant \rho_{m} \leqslant C \rho(x), \quad x \in S_{m},
$$

где $\rho_{m}=1+h(m+1 / 2)$, а $c$ и $C$ - положительные постоянные. Подчеркнем, что благодаря использованию вектор-функции $u$, продолженной нулем на $\{x: \pm z>1\}$, крайние ячейки не требуют отдельной обработки (см. п. 1). Далее индексы $m$ и \pm не пишем, а $x^{m}$ и $\rho_{m}$ обозначаем $x^{0}=\left(y^{0}, z^{0}\right)$ и $\rho_{0}$.

Поле $u$ представим на ячейке $S$ в виде

$$
u(x)=u^{\perp}(x)+d(x) a,
$$

где $u^{\perp} \in H^{1}(S)^{3}, a \in \mathbb{R}^{6}, z^{0}=h(m+1 / 2)$,

$$
\begin{gathered}
d(x)=\left(\begin{array}{cccccc}
1 & 0 & 0 & 0 & -\alpha\left(z-z^{0}\right) & \alpha\left(y_{2}-y_{2}^{0}\right) \\
0 & 1 & 0 & \alpha\left(z-z^{0}\right) & 0 & -\alpha\left(y_{1}-y_{1}^{0}\right) \\
0 & 0 & 1 & -\alpha\left(y_{2}-y_{2}^{0}\right) & \alpha\left(y_{1}-y_{1}^{0}\right) & 0
\end{array}\right) \\
a=d_{B}^{-1} \int_{B} d(x)^{t} u(x) d x \\
d_{B}=\int_{B} d(x)^{t} d(x) d x
\end{gathered}
$$

Обратим внимание на следующие факты. Во-первых, при любом столбце $a \in \mathbb{R}^{6}$ вектор $d(x) a$ является жестким смешением (транслящия + поворот), причем в силу $(1.5),(3.18)$ и $(3.17),(1.10) D\left(\nabla_{x}\right)^{t} d(x) a=0$ и

$$
E(u, u ; \Xi)=E\left(u^{\perp}, u^{\perp} ; \Xi\right)
$$

Во-вторых, столбцы матрицы $d$ линейно независимы, а (3.20) - симметрическая и положительно определенная матрища Грама размером $6 \times 6$, построенная по этим столбцам при помощи скалярного произведения в $L_{2}(B)^{3}$. В-третьих,

$$
\begin{aligned}
\int_{B} d(x)^{t} u^{\perp}(x) d x & =\int_{B} d(x)^{t} u(x) d x-\int_{B} d(x)^{t} d(x) d x a \\
& =\int_{B} d(x)^{t} u(x) d x-d_{B} d_{B}^{-1} \int_{B} d(x)^{t} u(x) d x=0
\end{aligned}
$$

а значит, сужение $u^{\perp}$ на $B$ принадлежит подпространству, имеющему тривиальное пересечение с линеалом жестких смешений, и справедливо неравенство Корна

$$
\left\|u^{\perp} \mid\right\|_{B}^{2} \leqslant c E\left(u^{\perp}, u^{\perp} ; B\right)
$$


(доказательство этого неравенства можно найти, например, в [34, § 2]; в дополнение к нему нужно сделать замену координат $x \mapsto \tilde{x}=h^{-1}\left(x-x^{m}\right)$, учитьвающую малый параметр $h$ ).

Принимая во внимание предположение іiі) и формулы (3.21), (3.22), заключаем, что

$$
\|\left.\left|u^{\perp}\right|\right|_{S} ^{2} \leqslant c\left(E\left(u^{\perp}, u^{\perp} ; S\right)+\left\|u^{\perp} \mid\right\|_{B}^{2}\right) \leqslant C E\left(u^{\perp}, u^{\perp} ; S\right)=C E(u, u ; S) .
$$

Кроме того, поскольку $h^{2} \rho(z)^{-2} \leqslant 1, \rho(z)^{-2} \leqslant h^{-2}$ и $h^{2} \rho(z)^{-4} \leqslant h^{-2}$, в силу определения норм $|\cdot ; S, \rho|$ и $\left.|||\cdot|\right|_{S}$ получаем

$$
\left|u^{\perp} ; S, \rho\right|^{2} \leqslant||\left|u^{\perp}\right|||_{S}^{2} \leqslant E(u, u ; S) .
$$

Итак, осталось оценить норму последнего слагаемого в (3.17). Отметим сначала, что несложные выкладки, используюшие (3.4), приводят к неравенству

$$
|d a ; S, \rho|^{2} \leqslant c h^{5} \rho_{0}^{-2}\left\{\rho_{0}^{-2} a_{1}^{2}+\rho_{0}^{-2} a_{2}^{2}+h^{-2} a_{3}^{2}+a_{4}^{2}+a_{5}^{2}+a_{6}^{2}\right\} .
$$

Обратимся теперь к формуле (3.19). Поскольку лемма 3.2 дает соотношение

$$
\left|u ; Q_{h}^{\prime}, \rho\right|^{2} \leqslant c\left(E\left(u, u ; Q_{h}\right)+E\left(u, u ; Q_{h}^{\prime}\right)\right) \leqslant 2 c E\left(u, u ; Q_{h}\right)
$$

и шар $B$ содержится в $Q_{h}^{\prime}$, в нашем распоряжении имеется норма $|u ; B, \rho|$, позволяющая оценить интеграл в правой части (3.19). Начало координат $\left(y-y^{0}, z-z^{0}\right)$ совмешено с центром шара $B$, и поэтому матрица (3.20) вычисляется легко:

$$
d_{B}=\frac{4 \pi}{3} h^{3} R_{ \pm}^{3} \operatorname{diag}\left\{1,1,1, \frac{1}{5} h^{2} R_{ \pm}^{2}, \frac{1}{5} h^{2} R_{ \pm}^{2}, \frac{1}{5} h^{2} R_{ \pm}^{2}\right\} .
$$

Первая пара компонент столбца $J=\left(J_{1}, \ldots, J_{6}\right)^{t}=\int_{B} d(x)^{t} u(x) d x$ удовлетворяет оценкам

$$
\left|J_{p}\right|^{2} \leqslant c \operatorname{mes}_{3}(B) \int_{B}\left|u_{p}\right|^{2} d x \leqslant \operatorname{ch} \rho_{0}^{4} \int_{B} \frac{h^{2}}{\rho^{4}}\left|u_{p}\right|^{2} d x \leqslant \operatorname{ch} \rho_{0}^{4}|u ; B, \rho|^{2}, \quad p=1,2
$$

(воспользовались (3.16)). Аналогично

$$
\left|J_{3}\right|^{2} \leqslant \operatorname{ch}^{3} \rho_{0}^{2} \int_{B} \rho^{-2}\left|u_{3}\right|^{2} d x \leqslant \operatorname{ch}^{3} \rho_{0}^{2}|u ; B, \rho|^{2} .
$$

Пусть $\mathscr{R}(x)=\left|y-y^{0}\right|^{2}+\left|z-z^{0}\right|^{2}-h^{2} R_{ \pm}^{2}$. Так как $\mathscr{R}=0$ на $\partial B$, то интегрирование по частям при учете (3.16) приводит к соотношению

$$
\begin{aligned}
\left|J_{6}\right|^{2} & =\frac{1}{2}\left|\int_{B}\left\{\left(y_{2}-y_{2}^{0}\right) u_{1}-\left(y_{1}-y_{1}^{0}\right) u_{2}\right\} d x\right|^{2} \\
& =\frac{1}{8}\left|\int_{B}\left\{u_{1} \frac{\partial \mathscr{R}}{\partial y_{2}}-u_{2} \frac{\partial \mathscr{R}}{\partial y_{2}}\right\} d x\right|^{2}=\frac{1}{8}\left|\int_{B} \mathscr{R}\left(\frac{\partial u_{2}}{\partial y_{1}}-\frac{\partial u_{1}}{\partial y_{2}}\right) d x\right|^{2} \\
& \leqslant \operatorname{ch}^{2} \rho_{0}^{2} \operatorname{mes}_{3}(B) \int_{B} \frac{h^{2}}{\rho^{2}}\left(\left|\frac{\partial u_{1}}{\partial y_{2}}\right|^{2}+\left|\frac{\partial u_{2}}{\partial y_{1}}\right|^{2}\right) d x \leqslant \operatorname{ch}^{2} \rho_{0}^{2}|u ; B, \rho|^{2} .
\end{aligned}
$$


Тот же трюк дает неравенства

$$
\left|J_{6-p}\right|^{2} \leqslant|u ; B, \rho|^{2}, \quad p=1,2 .
$$

Приведенная информация о $\left|J_{1}\right|, \ldots,\left|J_{6}\right|$ и формула (3.26) для $d_{B}$ позволяют оценить компоненты столбца $a=d_{B}^{-1} J$ и заключить, что правая часть (3.24) не превосходит $C|u ; B, \rho|^{2}$. Из сказанного и из (3.23) выводим, что

$$
\begin{aligned}
|u ; S, \rho|^{2} \leqslant & 2\left|u^{\perp} ; S, \rho\right|^{2}+2|d a ; S, \rho|^{2} \leqslant c(E(u, u ; S) \\
& \left.+|u ; B, \rho|^{2}\right) \leqslant C\left(E(u, u ; S)+\left|u ; Q_{h}^{\prime} \cap S, \rho\right|^{2}\right) .
\end{aligned}
$$

Просуммируем неравенства (3.27), которые справедливы для всех ячеек, составляюших стержень $\Omega_{h}$, и имеют общую постоянную $C$. Ввиду предположения ii)

$$
\left|u ; \Omega_{h}, \rho\right|^{2} \leqslant C\left\{E\left(u, u ; \Omega_{h}\right)+\left|u ; Q^{\prime+}, \rho\right|^{2}+\left|u ; Q^{\prime-}, \rho\right|^{2}\right\} .
$$

В силу (3.25) последние два слагаемых мажорируются величинами $c E\left(u, u ; Q_{h}^{ \pm}\right) \leqslant$ $c E\left(u, u ; \Omega_{h}\right)$, после чего (3.27) принимает вид искомого неравенства (3.5). Tеорема 3.1 доказана.

5. Одно утверждение о следах на боковой поверхности. Пусть вектор-функция $T \in C_{0}^{\infty}\left(\bar{\Omega}_{1}\right)^{2}$ такова, что

$$
\nu_{1}(\eta, z) T_{1}(\eta, z)+\nu_{2}(\eta, z) T_{2}(\eta, z)=1 \text { при } z \in(-1,1), \quad \eta \in \partial \omega(z) .
$$

Используя это равенство и интегрируя по частям на сечениях $\omega_{h}(z)$ стержня $\Omega_{h}$, получаем

$$
\begin{aligned}
\int_{\partial \omega_{h}(z)}|v(x)|^{2} d s_{y} & =\int_{\omega_{h}(z)} \nabla_{y} \cdot\left(T\left(h^{-1} y\right) v(x)^{2}\right) d y \\
& \leqslant c\left(h^{-1} \int_{\omega_{h}(z)}|v(x)|^{2} d y+\int_{\omega_{h}(z)}|v(x)|\left|\nabla_{y} v(x)\right| d y\right) \\
& \leqslant c h^{-1} \int_{\omega_{h}(z)}\left(|v(x)|^{2}+h^{2}\left|\nabla_{y} v(x)\right|^{2}\right) d y
\end{aligned}
$$

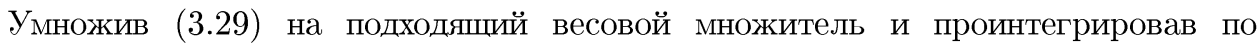
$z \in(-1,1)$, приходим к следуюшему утверждению.

ПРЕДЛОЖЕНИЕ 3.3. В условиях теоремы 3.1

$$
h^{3 / 2}\left\|\rho^{2} u_{i} ; L_{2}\left(\Gamma_{h}\right)\right\|+h^{1 / 2}\left\|\rho u_{3} ; L_{2}\left(\Gamma_{h}\right)\right\| \leqslant c|u|, \quad i=1,2
$$




\section{§ 4. Обоснование асимптотики}

1. Вспомогательная задача и ее решение. Пусть $\mathscr{U}$ - сумма первых четырех слагаемых из правой части (2.9). В силу (2.38) слагаемые $(2.16)$ и $(2.20)$ удовлетворяют (1.8). Вместе с тем для $\mathscr{U}^{(0)}$ и $\mathscr{U}^{(1)}$ (решения задач $(2.21)$ и (2.27)) условия Дирихле (1.8) выполняются разве лишш случайно. Поэтому, желая соблюсти (1.8), изменим вид асимптотического приближения, а именно умножим $w$ на срезающую функцию $\chi \in C_{0}^{\infty}(-1,1)$, т.е. положим $\mathbf{w}=\chi w$ и определим $\mathbf{U}^{(-2)}$, $\mathbf{U}^{(-1)}, \mathbf{U}^{(0)}$ формулами $(2.16),(2.20)$ и $(2.25)$ с заменой $w$ на $\mathbf{w}\left(\mathbf{U}^{(1)}\right.$ требует отдельного обсуждения). Поскольку $\chi=0$ вблизи торцов стержня $\Omega_{h}$, каждая вектор-функция (из трех построенных) удовлетворяет (1.8). Фиксируем срезку $\chi$ так, чтобы $\chi(z)=1$ при $|z|<1-2 h$ и $\chi(z)=0$ при $|z|>1-h$. По сушеству $\chi$ зависит от "быстрых" переменных $h^{-1}(z \mp 1)$ и верны оценки

$$
\left|\partial_{z}^{k} \chi(z)\right| \leqslant c_{k} h^{-k}, \quad k=0,1, \ldots
$$

однако в дальнейших вычислениях эту зависимость удобно считать медленной.

Слагаемое $\mathbf{U}^{(-1)}$ определяется как решение задачи, подобной (2.27), а условие ее разрешимости удается соблюсти за счет незначительного видоизменения представления (2.6) правой части исходной задачи (1.6)-(1.8). Предугадать это условие несложно: результируюшая задача для $\mathbf{w}$ получается в результате умножения системы (2.36) на $\chi$ и коммутирования дифференциальных операторов со срезкой. Иными словами, оно имеет вид

$$
\mathscr{D}\left(-\partial_{z}\right) \mathscr{M} \mathscr{D}\left(\partial_{z}\right) \mathbf{w}=\chi \mathscr{F}+\mathscr{F}^{1} \text { на }(1,-1),
$$

где $[A, B]=A B-B A-$ коммутатор операторов $A$ и $B$,

$$
\mathscr{F}^{1} \equiv\left(\mathscr{F}_{1}^{1}, \ldots, \mathscr{F}_{1}^{4}\right)^{t}=\left[\mathscr{D}\left(-\partial_{z}\right) \mathscr{M} \mathscr{D}\left(\partial_{z}\right), \chi\right] w
$$

Укажем изменения в (2.6), достаточные для того, чтобы уравнение (4.2) возникло в результате применения процедуры, описанной в $\S 2$. Во-первых, считаем, что $\tilde{f}=0$ и $\tilde{g}=0$. Во-вторых, заменим $f^{0}, g^{0}$ и $\bar{f}^{0}$ произведениями $\chi f^{0}=\mathbf{f}^{0}$, $\chi g^{0}=\mathbf{g}^{0}$ и $\chi \bar{f}^{0}=\overline{\mathbf{f}}^{0}$ (это действие приводит к появлению множителя $\chi$ при $\mathscr{F}$ в (4.2)). В-третьих, добавим к $\mathbf{f}^{0}, \mathbf{g}^{0}$ и $\overline{\mathbf{f}}^{0}$ выражения

$$
\begin{aligned}
\mathbf{f}^{1}(\eta, z) & =\sum_{q=1}^{4} \mathbf{H}_{i}(z) \Phi^{i}(\eta), \quad \mathbf{g}^{1}(\eta, z)=0, \\
\overline{\mathbf{f}}^{1}(z) & =|\omega|^{-1} \sum_{i=1}^{2} e^{i}\left\{\mathscr{F}_{i}^{1}(z)\right. \\
& \left.+\left(\left(\eta_{i}, f_{3}^{0}(\eta, z)\right)_{\omega(z)}+\left(\eta_{i}, g_{3}^{0}(\eta, z)\right)_{\partial \omega(z)}\right) \partial_{z} \chi(z)\right\},
\end{aligned}
$$


где $\Phi^{q}$ - жесткие смешения (2.14), а столбец $\mathbf{H}=\left(\mathbf{H}_{1}, \ldots, \mathbf{H}_{4}\right)^{t}$ является решением алгебраической системы

$$
\mathbf{K}(z) \mathbf{H}(z)=\left(0,0, \mathscr{F}_{3}^{1}(z), \mathscr{F}_{4}^{1}(z)\right)^{t}
$$

с симметрической и положительно определенной $(4 \times 4)$-матрищей Грама $\mathbf{K}=$ $\left(\left(\Phi^{q}, \Phi^{p}\right)_{\omega(z)}\right)_{p, q=1}^{4}$.

Приведем пояснения к формулам (4.4)-(4.6). В силу (4.6) вектор-функции $\mathbf{f}^{1}$, $\mathbf{g}^{1}$ и $\overline{\mathbf{f}}^{1}$ удовлетворяют требованиям $(2.7),(2.8)$ и формируют в результирующей задаче для w правую часть $\mathscr{F}_{3}^{1}$. Кроме того, последнее слагаемое из фигурных скобок в (4.5) учитывает наличие дифференцирования $\partial_{z}$ в представлении $(2.35)$ для $\mathscr{F}_{1}$ и $\mathscr{F}_{2}$ (возникает дополнительный коммутатор). Наконец, каждая из величин в (4.4) и (4.5) обрашается в нуль при $|z|>1-h$ и $|z|<1-2 h$ (поскольку содержит производные срезающей функции $\chi$ ).

Итак, в силу (4.2) выполнены условия разрешимости задачи

$$
\begin{aligned}
L^{0} \mathbf{U}^{(1)}= & \mathbb{D}_{\eta} A \mathbb{D}_{z}^{t} \mathbf{U}^{(0)}+\mathbb{D}_{z} A\left\{\mathbb{D}_{\eta}^{t} \mathbf{U}^{(0)}+\mathbb{D}_{z}^{t} \mathbf{U}^{(-1)}\right\}+\mathbf{f}^{0}+\mathbf{f}^{1} \text { в } \omega(z), \\
B^{0} \mathbf{U}^{(1)}= & -\mathbb{D}_{\nu} A \mathbb{D}_{z}^{t} \mathbf{U}^{(0)}-\nu_{0} \mathbb{D}_{1} A\left\{\mathbb{D}_{\eta}^{t} \mathbf{U}^{(0)}+\mathbb{D}_{z}^{t} \mathbf{U}^{(-1)}\right\} \\
& +\mathbf{g}^{0}+\mathbf{g}^{1} \text { на } \partial \omega(z),
\end{aligned}
$$

и вектор-функция $\mathbf{U}^{(1)}$ построена. Сумму $\mathbf{U}=h^{-2} \mathbf{U}^{(-2)}+\cdots+h^{1} \mathbf{U}^{(1)}$ назовем вспомогательным решением. Вычислим невязку этого решения в уравнении (1.6) с измененной правой частью. Учитывая расщепление (2.4) оператора $L$, имеем

$$
\begin{aligned}
L \mathbf{U}- & h^{-1}\left(\mathbf{f}^{0}+\mathbf{f}^{1}\right)-h^{0}\left(\overline{\mathbf{f}}^{0}+\overline{\mathbf{f}}^{1}\right)=h^{-4} L^{0} \mathbf{U}^{(-2)}+h^{-3}\left(L^{0} \mathbf{U}^{(-1)}+L^{1} \mathbf{U}^{(-2)}\right) \\
& +h^{-2}\left(L^{0} \mathbf{U}^{(0)}+L^{1} \mathbf{U}^{(-1)}+L^{2} \mathbf{U}^{(-2)}\right) \\
& +h^{-1}\left(L^{0} \mathbf{U}^{(1)}+L^{1} \mathbf{U}^{(0)}+L^{2} \mathbf{U}^{(-1)}-\mathbf{f}^{0}-\mathbf{f}^{1}\right) \\
& +h^{0}\left(L^{1} \mathbf{U}^{(1)}+L^{2} \mathbf{U}^{(0)}+\overline{\mathbf{f}}^{0}+\overline{\mathbf{f}}^{1}\right)+h^{1} L^{2} \mathbf{U}^{(1)}
\end{aligned}
$$

Вспоминая структуру краевых задач для $\mathbf{U}^{(j)}$ (см. $(2.10),(2.11)$, а также (4.7)), обнаруживаем, что в (4.8) множители при $h^{-4}, \ldots, h^{-1}$ равны нулю. Введем краткие обозначения для последних двух слагаемых и перепишем (4.8) так:

$$
L \mathbf{U}-h^{-1}\left(\mathbf{f}^{0}+\mathbf{f}^{1}\right)-h^{0}\left(\overline{\mathbf{f}}^{0}+\overline{\mathbf{f}}^{1}\right)=h^{0} \mathbf{F}^{0}-h^{1} \partial_{z} \mathbf{F}^{\prime},
$$

здесь $\mathbf{F}^{\prime}=\mathbb{D}_{1} A \mathbb{D}_{z}^{t} \mathbf{U}^{(1)}$ (поскольку $L^{2}=-\partial_{z} \mathbb{D}_{1} A \mathbb{D}_{z}^{t} ;$ см. (2.5)). Аналогично обрабатывается невязка, порожденная $\mathbf{U}$ в краевом условии (1.7) с правой частью $\mathrm{g}^{0}+\mathbf{g}^{1}$. Принимая во внимание $(2.3)$ и $(2.4)$, находим, что

$$
B \mathbf{U}-\mathbf{g}^{0}-\mathbf{g}^{1}=h^{1} \mathbf{G}^{0}+h^{0} N^{-1 / 2} \nu_{0} \mathbf{F}^{\prime}+\left(N^{-1 / 2}-1\right)\left(\mathbf{G}^{0}+\mathbf{g}^{0}+\mathbf{g}^{1}\right)
$$

(см. формулу для $B^{2}$ в (2.5)). Отметим, что множитель $N(h, x)^{-1 / 2}-1=O\left(h^{2}\right)$ мал,

$$
\mathbf{G}^{0}=\mathbb{D}_{\nu} A \mathbb{D}_{z}^{t} \mathbf{U}^{(0)}+\nu_{0} \mathbb{D}_{1} A\left(\mathbb{D}_{\eta}^{t} \mathbf{U}^{(0)}+\mathbb{D}_{z}^{t} \mathbf{U}^{(-1)}\right)
$$

и в соответствии с п. $5 \S 2$

$$
\left(\mathbf{F}^{0}, e^{i}\right)_{\omega(z)}+\left(\mathbf{G}^{0}, e^{i}\right)_{\partial \omega(z)}=0, \quad z \in(-1,1), \quad i=1,2 .
$$

Наконец, U удовлетворяет условиям Дирихле (1.8). 
2. Сравнение вспомогательного и асимптотического решений. Оценка разности $\mathscr{U}-\mathbf{U}$, полученная в данном пункте, позволит во всех дальнейших рассмотрениях иметь дело только со вспомогательным решением.

Сначала уточним свойства слагаемых в разложениях (2.6). Именно, будем считать, что

$$
\begin{aligned}
f^{0} \in H^{1}((-1,1) & \left.\ni z \longmapsto L_{2}(\omega(z))^{3}\right), \\
g^{0} \in H^{1}((-1,1) & \left.\ni z \longmapsto H^{1 / 2}(\partial \omega(z))^{3}\right), \\
\bar{f}^{0} & \in L_{2}(-1,1)^{3} .
\end{aligned}
$$

Более того, нормы в пространствах $H^{1}(\ldots)$ по определению равны соответственно

$$
\left\|f^{0} \circ \varkappa^{-1} ; H^{1}\left((-1,1) \rightarrow L_{2}(\omega)^{3}\right)\right\|, \quad\left\|g^{0} \circ \varkappa^{-1} ; H^{1}\left((-1,1) \rightarrow H^{1 / 2}(\partial \omega)^{3}\right)\right\| ;
$$

здесь $\varkappa$ - диффеоморфизм, упомянутый в п. $1 \S 1$, и $H^{1}(\Delta \rightarrow \mathfrak{H})$ - пространство абстрактных функций на отрезке $\Delta$ со значениями в гильбертовом пространстве $\mathfrak{H}$,

$$
\left\|Z ; H^{1}(\Delta \rightarrow \mathfrak{H})\right\|=\left(\int_{\Delta}\left(\|Z ; \mathfrak{H}\|^{2}+\left\|\partial_{z} Z ; \mathfrak{H}\right\|^{2}\right) d z\right)^{1 / 2}
$$

Сумму норм функций (4.13) в указанных пространствах обозначим $\mathscr{N}_{0}$. В силу формул $(2.31),(2.35)$ и теоремы 2.7 , в которой $l=1$, справедливо соотношение

$$
\left\|w ; H^{4}(-1,1)^{2} \times H^{3}(-1,1)^{2}\right\| \leqslant c\left\|\mathscr{F} ; L_{2}(-1,1)^{2} \times H^{1}(-1,1)^{2}\right\| \leqslant C \mathscr{N}_{0}
$$

краткая запись которого такова: $\mathscr{N}_{w} \leqslant \mathscr{N}_{\mathscr{F}} \leqslant C \mathscr{N}_{0}$.

ПРЕДЛОЖЕНИЕ 4.1. Вектор-функиии $\mathscr{U}$ и $\mathbf{U}$ связаны соотношением

$$
\left|\mathscr{U}-\mathbf{U} ; \Omega_{h}, \rho\right| \leqslant C_{\delta} h^{(1-\delta) / 2} \mathscr{N}_{0}
$$

в котором $\delta>0$ - произвольное число, $|\cdot|$ - выражение $(3.4)$, постоянная $C_{\delta}$ зависит от $\delta$, но не от $w$ и $h$.

ДоКАЗАТЕЛЬСТво. Согласно определению $\mathscr{U}$ и $\mathbf{U}$ достаточно установить оценки

$$
h^{k}\left|\mathscr{U}^{(k)}-\mathbf{U}^{(k)} ; \Omega_{h}, \rho\right| \leqslant c_{\delta} h^{(1-\delta) / 2} \mathscr{N}_{0},
$$

где $k=-2,-1,0,1$. Среди первой тройки индексов рассмотрим представительный случай $k=-1$. В силу (2.20)

$$
\mathscr{U}^{(-1)}-\mathbf{U}^{(-1)}=(1-\chi)\left(w_{3} e^{3}+w_{4} \Phi^{4}-e^{3} \sum_{i=1}^{2} \eta_{i} \partial_{z} w_{i}\right)-e^{3} \sum_{i=1}^{2} \eta_{i} w_{i} \partial_{z} \chi
$$


Применяя различные варианты одномерного неравенства Харди (см. [39]), находим, что при любом $\delta>0$ в соответствии с $(2.38)$

$$
\begin{aligned}
& \int_{-1}^{1}(1-|z|)^{\delta-3}\left\{\left|w_{i+2}\right|^{2}+\left|\partial_{z} w_{i}\right|^{2}+(1-|z|)^{-2}\left|w_{i}\right|^{2} d z\right\} \\
& \quad \leqslant c_{\delta} \int_{-1}^{1}(1-|z|)^{\delta-1}\left\{\left|\partial_{z} w_{i+2}\right|^{2}+\left|\partial_{z}^{2} w_{i}\right|^{2}\right\} d z \\
& \quad \leqslant c_{\delta} \int_{-1}^{1}(1-|z|)^{\delta+1}\left\{\left|\partial_{z} w_{i+2}\right|^{2}+\left|\partial_{z}^{2} w_{i+2}\right|^{2}+\left|\partial_{z}^{2} w_{i}\right|^{2}+\left|\partial_{z}^{3} w_{i}\right|^{2}\right\} d z \\
& \quad \leqslant c_{\delta} \mathscr{N}_{w}^{2}, \quad i=1,2 .
\end{aligned}
$$

Заметим, что носители функций $1-\chi$ и $\partial_{z} \chi$ содержатся во множестве $\Delta(h)=$ $[-1,2 h-1] \cup[1-2 h, 1]$, на котором $h \leqslant \rho(z) \leqslant 3 h$ и $(1-|z|)^{-1} \geqslant(2 h)^{-1}$. Теперь в соответствии с $(4.17)$ и $(3.4),(4.1)$ получаем

$$
\begin{aligned}
h^{-2} & \left|\mathcal{U}^{(-1)}-\mathbf{U}^{(-1)} ; \Omega_{h}, \rho\right|^{2} \leqslant c h^{-2} \sum_{i=1}^{2} \int_{\Delta(h)} \operatorname{mes}_{2}\left(\omega_{h}(z)\right) \\
& \times\left\{h^{-4}\left|w_{i}\right|^{2}+h^{-2}\left(\left|\partial_{z} w_{i}\right|^{2}+\left|w_{i+2}\right|^{2}\right)+h^{0}\left(\left|\partial_{z}^{2} w_{i}\right|^{2}+\left|\partial_{z} w_{i+2}\right|^{2}\right)\right\} d z \\
\leqslant & c \sum_{i=1}^{2} h^{5-\delta-4} \int_{\Delta(h)}(1-|z|)^{\delta-5}\left\{\left|w_{i}\right|^{2}+(1-|z|)^{2}\left(\left|\partial_{z} w_{i}\right|^{2}+\left|w_{i+2}\right|^{2}\right)\right. \\
& \left.+(1-|z|)^{4}\left(\left|\partial_{z}^{2} w_{i}\right|^{2}+\left|\partial_{z} w_{i+2}\right|^{2}\right)\right\} d z \leqslant c_{\delta} h^{1-\delta} \mathcal{N}_{w}^{2}
\end{aligned}
$$

причем $\omega_{h}(z)=\left\{y: h^{-1} y \in \omega(z)\right\}$ - сечение стержня, обладающее площадью $O\left(h^{2}\right)$.

Формулы (4.18) и (4.14) приводят к неравенству (4.16) при $k=-1$. Поскольку известен явный вид $\mathscr{U}^{(-2)}, \mathbf{U}^{(-2)}$ и $\mathscr{U}^{(0)}, \mathbf{U}^{(0)}$ (см. (2.16) и $(2.25)$ ), вывод оценок (4.16) при $k=-2$ и $k=0$ столь же прост.

Обратимся к случаю $k=1$. В силу (4.7) и (2.27) разность $\mathscr{R}^{(1)}=\mathscr{U}^{(1)}-\mathbf{U}^{(1)}$ является решением задачи $(2.12)$, в которой

$$
\begin{aligned}
\mathscr{F}= & (1-\chi)\left\{\mathbb{D}_{\eta} A \mathbb{D}_{z}^{t \mathscr{V}}+\mathbb{D}_{z} A\left(\mathbb{D}_{\eta}^{t \mathscr{V}}+\mathscr{Y}\right)\right\} \mathscr{D}\left(\partial_{z}\right) w \\
& +\mathscr{K}^{1} w+(1-\chi) f^{0}-\mathbf{f}^{1}, \\
\mathscr{G}= & -(1-\chi)\left\{\mathbb{D}_{\nu} A \mathbb{D}_{z}^{t} \mathscr{V}+\nu_{0} \mathbb{D}_{1} A\left(\mathbb{D}_{\eta}^{t} \mathscr{V}+\mathscr{Y}\right)\right\} \mathscr{D}\left(\partial_{z}\right) w \\
& +\mathscr{K}^{0} w+(1-\chi) g^{0}-\mathbf{g}^{1},
\end{aligned}
$$

где $\mathbf{f}^{1}$ и $\mathbf{g}^{1}=0$ взяты из (4.4), а $\mathscr{K}^{1}$ и $\mathscr{K}^{0}$ - коммутаторы, образовавшиеся в результате вынесения срезки $\chi$ за дифференциальные операторы $\mathbb{D}_{z}$ и $\mathscr{D}\left(\partial_{z}\right)$. Иными словами,

$$
\begin{aligned}
\mathscr{K}^{p}\left(\eta, z, \partial_{z}\right) w(z)= & \sum_{i=1}^{2}\left\{\sum_{s=0}^{3} h^{s-3} k_{i, s}(\eta, z) \partial_{z}^{s} w_{i}(z)\right. \\
& \left.+\sum_{s=0}^{2} h^{s-2} k_{i+2, s}(\eta, z) \partial_{z}^{s} w_{i+2}(z)\right\}
\end{aligned}
$$


причем в силу (4.1)

$$
\left|k_{q, s}(\eta, z)\right|+h\left|\partial_{z} k_{q, s}(\eta, z)\right| \leqslant \text { const } .
$$

Вектор-функции (4.19) аннулируются вне множества $\Upsilon(h)=\left\{x \in \bar{\Omega}_{h}: 1 \geqslant|z| \geqslant\right.$ $1-2 h\}$. Подчеркнем, что $h \leqslant \rho \leqslant 3 h$ на $\Upsilon(h)$. Теперь, вычисляя нужную норму, применим оценку, указанную в предложении 2.1, и получим

$$
\begin{aligned}
h^{2} \mid \mathscr{U}^{(1)} & -\mathbf{U}^{(1)} ; \Upsilon(h),\left.\rho\right|^{2} \leqslant \\
\leqslant & c h^{2} \int_{\Delta(h)}\left(h^{-2}\left\|\mathscr{R}^{(1)} ; H^{1}(\omega(z))\right\|^{2}+\left\|\partial_{z} \mathscr{R}^{(1)} ; H^{1}(\omega(z))\right\|^{2}\right) h^{2} d z \\
\leqslant & c h^{4}\left\{\int _ { \Delta ( h ) } \left(h^{-2}\left\|\left(f^{0}, g^{0}\right) ; L_{2}(\omega(z)) \times L_{2}(\partial \omega(z))\right\|^{2}+\right.\right. \\
& \left.+\left\|\left(\partial_{z} f^{0}, \partial_{z} g^{0}\right) ; L_{2}(\omega(z)) \times L_{2}(\partial \omega(z))\right\|^{2}\right) d z \\
& +\int_{\Delta(h)}\left(h^{-2}\left\|\mathbf{f}^{1} ; L_{2}(\omega(z))\right\|^{2}+\left\|\partial_{z} \mathbf{f}^{1} ; L_{2}(\omega(z))\right\|^{2}\right) d z \\
& +\sum_{i=1}^{2}\left(\sum_{s=0}^{4} h^{2(s-4)} \int_{\Delta(h)}\left|\partial_{z}^{2} w_{i}\right|^{2} d z\right. \\
& \left.\left.+\sum_{s=0}^{3} h^{2(s-3)} \int_{\Delta(h)}\left|\partial_{z}^{2} w_{i+2}\right|^{2} d z\right)\right\}
\end{aligned}
$$

(не забыли о дополнительном множителе $\operatorname{mes}_{2}\left(\omega_{h}(z)\right)=O\left(h^{2}\right)$ ). Мажоранта $c h^{-2} \mathcal{N}_{0}^{2}$ для первого интеграла $I_{1}$ в правой части (4.21) находится посредством расширения области интегрирования до интервала $(-1,1)$. В согласии с $(4.4),(4.6)$ и (4.3) второй интеграл $I_{2}$ обрабатывается следующим образом:

$$
\begin{aligned}
I_{2} \leqslant & \int_{\Delta(h)}\left(\left\|\mathbf{K}(z)^{-1}\right\|^{2}+\left\|\partial_{z} \mathbf{K}(z)^{-1}\right\|^{2}\right) \sum_{q=3}^{4}\left(h^{-2}\left\|\mathscr{F}_{q}^{1} ; L_{2}(\omega(z))\right\|^{2}\right. \\
& \left.+\left\|\partial_{z} \mathscr{F}_{q}^{1} ; L_{2}(\omega(z))\right\|^{2}\right) d z \\
\leqslant & c h^{-2} \int_{\Delta(h)}\left|\left[\mathscr{D}\left(-\partial_{z}\right) \mathscr{M} \mathscr{D}\left(\partial_{z}\right), \chi(z)\right] w(z)\right|^{2} d z \\
\leqslant & c h^{-2} \sum_{i=1}^{2}\left(\sum_{s=0}^{3} h^{2(s-3)} \int_{\Delta(h)}\left|\partial_{z}^{s} w_{i}(z)\right|^{2} d z\right. \\
& \left.+\sum_{s=0}^{2} h^{2(s-2)} \int_{\Delta(h)}\left|\partial_{z}^{s} w_{i+2}(z)\right|^{2} d z\right) .
\end{aligned}
$$

Последнее выражение не превосходит $c \Sigma$, где $\Sigma$ - сумма по $i=1,2$ из фигурных скобок в (4.21). Таким образом, оценивание правой части (4.21) заканчивается аналогично (4.18): при помоши (4.17) проверяем, что

$$
h^{4} \Sigma \leqslant c_{\delta} h^{4} \sum_{i=1}^{2}\left\{\sum_{s=0}^{2} h^{2(s-4)+5-\delta-2 s} \int_{\Delta(h)}(1-|z|)^{\delta-5+2 s}\left|\partial_{z}^{s} w_{i}(z)\right|^{2} d z\right.
$$




$$
\begin{aligned}
& +\sum_{s=3}^{4} h^{2(s-4)} \int_{\Delta(h)}\left|\partial_{z}^{s} w_{i}(z)\right|^{2} d z \\
& +\sum_{s=0}^{1} h^{2(s-3)+3-\delta-2 s} \int_{\Delta(h)}(1-|z|)^{\delta-3+2 s}\left|\partial_{z}^{s} w_{i+2}(z)\right|^{2} d z \\
& \left.+\sum_{s=2}^{3} h^{2(s-3)} \int_{\Delta(h)}\left|\partial_{z}^{s} w_{i+2}(z)\right|^{2} d z\right\} \leqslant C_{\delta} h^{1-\delta} \mathcal{N}_{w}^{2} .
\end{aligned}
$$

Итак, оценки (4.16), а значит, и само неравенство (4.15) установлены.

3. Промежуточная оценка асимптотического остатка. Фиксируем некоторое $\delta>0$ в неравенстве (4.15). Малость остаточных членов $\tilde{f}$ и $\tilde{g}$ в представлениях (2.6) выразим соотношением

$$
\begin{aligned}
& h^{-1} \sum_{i=1}^{2}\left(\left\|\rho^{2} \tilde{f}_{i} ; L_{2}\left(\Omega_{h}\right)\right\|+h^{1 / 2}\left\|\rho^{2} \tilde{g}_{i} ; L_{2}\left(\Gamma_{h}\right)\right\|\right)+ \\
& \quad+\left\|\rho \tilde{f}_{3} ; L_{2}\left(\Omega_{h}\right)\right\|+h^{1 / 2}\left\|\rho \tilde{g}_{3} ; L_{2}\left(\Gamma_{h}\right)\right\|=h^{(1-\delta) / 2} \widetilde{\mathcal{N}}_{\delta},
\end{aligned}
$$

считая, что $\widetilde{\mathcal{N}}_{\delta}-$ "величина порядка единищы".

Согласно (1.6)-(1.8), (2.6) и (4.9), (4.10) разность $\mathbf{R}=u-\mathbf{U}$ удовлетворяет задаче

$$
\begin{gathered}
L \mathbf{R}=\tilde{f}+\mathbf{F}-\mathbf{F}^{0}+h \partial_{z} \mathbf{F}^{\prime} \text { в } \Omega_{h}, \\
B \mathbf{R}=\tilde{g}+\mathbf{G}-\mathbf{G}^{0}-N^{-1 / 2} \nu_{0} \mathbf{F}^{\prime} \text { на } \Gamma_{h}, \\
\mathbf{R}=0 \text { на } \omega_{h}( \pm 1),
\end{gathered}
$$

где $\mathbf{F}^{0}, \mathbf{G}^{0}$ и $\mathbf{F}^{\prime}$ определены в $(4.9),(4.11)$ и

$$
\begin{aligned}
& \mathbf{F}=h^{-1}(1-\chi) f^{0}+h^{-1} \mathbf{f}^{1}+(1-\chi) \bar{f}^{0}+\overline{\mathbf{f}}^{1}, \\
& \mathbf{G}=(1-\chi) g^{0}+\left(1-N^{-1 / 2}\right)\left(\mathbf{G}+\chi g^{0}\right) .
\end{aligned}
$$

Подставим $\mathbf{R}$ в формулу Грина (1.11) на обе позищии. Пользуясь (4.25) и "перебрасывая" производную $\partial_{z} \mathbf{c} \mathbf{F}^{\prime}$ на $\mathbf{R}$ при учете (2.2), получаем равенство

$$
\begin{aligned}
E\left(\mathbf{R}, \mathbf{R} ; \Omega_{h}\right)= & (\tilde{f}, \mathbf{R})_{\Omega_{h}}+(\tilde{g}, \mathbf{R})_{\Gamma_{h}}+(\mathbf{F}, \mathbf{R})_{\Omega_{h}}+(\mathbf{G}, \mathbf{R})_{\Gamma_{h}} \\
& -\left(\mathbf{F}^{0}, \mathbf{R}\right)_{\Omega_{h}}-\left(h \mathbf{G}^{0}, \mathbf{R}\right)_{\Gamma_{h}}-h\left(\mathbf{F}^{\prime}, \partial_{z} \mathbf{R}\right)_{\Omega_{h}}
\end{aligned}
$$


ЗАмЕчАнИЕ 4.2. Предположение (4.13) обеспечивает включение $w$ и $\mathbf{w}$ в пространство $H^{4}(-1,1)^{2} \times H^{3}(-1,1)^{2}$. Вместе с тем, вектор-функции $\mathscr{U}$ и $\mathbf{U}$ принадлежат $H^{1}\left(\Omega_{h}\right)^{3}$, а каждое из слагаемых $\mathbf{F}, \mathbf{F}^{0}, \mathbf{F}^{\prime}$ и $\mathbf{G}, \mathbf{G}^{0}, \mathbf{G}^{\prime}$ в (4.25) содержится в $L_{2}\left(\Omega_{h}\right)^{3}$ и $L_{2}\left(\Gamma_{h}\right)^{3}$ соответственно. Таким образом, задача $(4.25)$ нуждается в слабой постановке (как интегральное тождество, получающееся, если $\mathbf{R}$ на вторых позициях в (4.27) заменить произвольной пробной функцией $v \in \stackrel{\circ}{H^{1}}\left(\Omega_{h}\right)^{3}$, подчиненной (1.8)). Поскольку для вывода дальнейших оценок требуется только равенство (4.27), мы избегаем обсуждать задачу (4.24) более подробно.

Теорема 3.1 дает оценку снизу для левой части(4.27) через величину $|\mathbf{R}|^{2}(c-$ положительная постоянная). Теперь нужно оценить слагаемые справа в (4.27). Благодаря (4.24) и (3.4) сразу же обнаруживаем, что

$$
\left|(\tilde{f}, \mathbf{R})_{\Omega_{h}}+(\tilde{g}, \mathbf{R})_{\Gamma_{h}}\right| \leqslant c h^{(1-\delta) / 2 \widetilde{N_{\delta}}}|\mathbf{R}|
$$

Итак, для доказательства неравенства

$$
c_{A}|\mathbf{R}|^{2} \leqslant E\left(\mathbf{R}, \mathbf{R} ; \Omega_{h}\right) \leqslant c h^{(1-\delta) / 2}\left(\widetilde{\mathscr{N}}_{\delta}+\mathscr{N}_{0}\right)|\mathbf{R}|
$$

достаточно проверить такие оценки:

$$
\begin{aligned}
\left|(\mathbf{F}, \mathbf{R})_{\Omega_{h}}+(\mathbf{G}, \mathbf{R})_{\Gamma_{h}}\right| & \leqslant c h^{(1-\delta) / 2} \mathscr{N}_{0}|\mathbf{R}| \\
\left|\left(\mathbf{F}^{0}, \mathbf{R}\right)_{\Omega_{h}}+\left(h \mathbf{G}^{0}, \mathbf{R}\right)_{\Gamma_{h}}\right| & \leqslant c h^{(1-\delta) / 2} \mathscr{N}_{0}|\mathbf{R}| \\
\left\|\rho \mathbf{F}^{\prime} ; L_{2}\left(\Omega_{h}\right)\right\| & \leqslant c h^{(1-\delta) / 2} \mathscr{N}_{0}
\end{aligned}
$$

Формула (4.31) написана при учете (3.4); с нее и начнем проверку. Так как $\mathbf{F}^{\prime}=\mathbb{D}_{1} A \mathbb{D}_{z}^{t} \mathbf{U}^{(1)}$, имеем

$$
\left\|\rho \mathbf{F}^{\prime} ; L_{2}\left(\Omega_{h}\right)\right\|^{2} \leqslant c\left\|\rho \partial_{z} \mathbf{U}^{(1)} ; L_{2}\left(\Omega_{h}\right)\right\|^{2} .
$$

Обрашаясь к задаче (4.7) и формулам (2.25), (2.20), видим, что последнее выражение не превосходит

$$
\begin{aligned}
& c h^{2}\left\{\left\|\rho \mathbf{w} ; H^{4}(-1,1)^{2} \times H^{3}(-1,1)^{2}\right\|^{2}+\int_{\Delta(h)} \rho^{2}\left\|\partial_{z} \mathbf{f}^{1} ; L_{2}(\omega(z))\right\|^{2} d z\right. \\
&\left.\quad+\int_{-1}^{1} \rho^{2}\left\|\left(\partial_{z} \mathbf{f}^{0}, \partial_{z} \mathbf{g}^{0}\right) ; L_{2}(\omega(z)) \times L_{2}\left(\partial_{z} \omega(z)\right)\right\|^{2} d z\right\} \\
& \leqslant h^{2}\left\{\left\|w ; H^{4}(-1,1)^{2} \times H^{3}(-1,1)^{2}\right\|^{2}+h^{2}\left(\Sigma+I_{2}\right)+\mathscr{N}_{0}^{2}\right\} \\
& \leqslant c\left(h^{2} \mathscr{N}_{w}^{2}+h^{1-\delta} \mathscr{N}_{w}^{2}+h^{2} \mathscr{N}_{0}^{2}\right) \leqslant C h^{1-\delta} \mathscr{N}_{0}^{2} .
\end{aligned}
$$

Поясним выкладки. Вычисляя норму $\mathbf{w}=\chi w$, произвели коммутирование со срезкой $\chi$, вызвавшее появление величины $\Sigma$, уже фигурировавшей в (4.21). Интеграл 
по $\Delta(h)$ сравнили с аналогичным интегралом $I_{2}(4.21)$. При рассмотрении интеграла, содержащего $\mathbf{f}^{0}=\chi f^{0}$ и $\mathbf{g}^{0}=\chi g^{0}$, учли неравенство $\rho \leqslant$ const и включения (4.13). Обший множитель $h^{2}$ связан с плошадью сечения $\omega_{h}(z)$, а дополнительные множители $h^{2}$ при $\Sigma$ и $I_{2}-$ с весом $\rho$, равным $O(h)$ на $\Delta(h)$.

Убедившись в справедливости (4.31), приступим к проверке (4.30). Вспоминая условия ортогональности (4.12), имеем

$$
\left(\mathbf{F}_{i}^{0}, \mathbf{R}_{i}\right)_{\Omega_{h}}+\left(h \mathbf{G}_{i}^{0}, \mathbf{R}_{i}\right)_{\Gamma_{h}}=\left(\mathbf{F}_{i}^{0}, \mathbf{R}_{i}^{\perp}\right)_{\Omega_{h}}+\left(h \mathbf{G}_{i}^{0}, \mathbf{R}_{i}^{\perp}\right)_{\Gamma_{h}}, \quad i=1,2
$$

(множитель $h$ исчезает при переходе к быстрым переменным); здесь

$$
\mathbf{R}_{i}^{\perp}(y, z)=\mathbf{R}_{i}(y, z)-\left|\omega_{h}(z)\right|^{-1} \int_{\omega_{h}(z)} \mathbf{R}_{i}(y, z) d y,
$$

причем в силу неравенства Пуанкаре, примененного на каждом из сечений стержня,

$$
\begin{aligned}
|\mathbf{R}|^{2} & \geqslant\left\|h \rho^{-1} \nabla_{y} \mathbf{R}_{i} ; L_{2}\left(\Omega_{h}\right)\right\|^{2} \\
& =h^{2}\left\|\rho^{-1} \nabla_{y} \mathbf{R}_{i}^{\perp} ; L_{2}\left(\Omega_{h}\right)\right\|^{2} \geqslant c\left\|\rho^{-1} \mathbf{R}_{i}^{\perp} ; L_{2}\left(\Omega_{h}\right)\right\|^{2}
\end{aligned}
$$

и при учете $(3.29)$

$$
|\mathbf{R}|^{2} \geqslant \operatorname{ch}\left\|\rho^{-1} \mathbf{R}_{i}^{\perp} ; L_{2}\left(\Gamma_{h}\right)\right\|^{2} .
$$

Случай $i=3$ не требует отдельных рассмотрений. В итоге

$$
\left|\left(\mathbf{F}^{0}, \mathbf{R}\right)_{\Omega_{h}}+\left(h \mathbf{G}^{0}, \mathbf{R}\right)_{\Gamma_{h}}\right| \leqslant c|\mathbf{R}|\left\{\left\|\rho \mathbf{F}^{0} ; L_{2}\left(\Omega_{h}\right)\right\|^{2}+h\left\|\rho \mathbf{G}^{0} ; L_{2}\left(\Gamma_{h}\right)\right\|^{2}\right\}^{1 / 2} .
$$

Изучим выражение $J$ из фигурных скобок. Явные формулы для $\mathbf{G}^{0}$ и $\mathbf{F}^{0}$ (см. (4.11) и ср. (4.9) с (4.8)) показывают, что

$$
\begin{aligned}
J \leqslant & c h^{2}\left\{\left\|\rho \mathbf{w} ; H^{4}(-1,1)^{2} \times H^{3}(-1,1)^{2}\right\|^{2}\right. \\
& +\int_{\Delta(h)} \rho^{2}\left\|\partial_{z} \mathbf{f}^{1} ; L_{2}(\omega(z))\right\|^{2} d z \\
& +\int_{-1}^{1} \rho^{2}\left\|\left(\partial_{z} \mathbf{f}^{0}, \partial_{z} \mathbf{g}^{0}\right) ; L_{2}(\omega(z)) \times L_{2}\left(\partial_{z} \omega(z)\right)\right\|^{2} d z \\
& \left.+\int_{-1}^{1} \rho^{2} \chi^{2}\left|\bar{f}^{0}\right|^{2} d z+\int_{\Delta(h)} \rho^{2}\left|\overline{\mathbf{f}}^{1}\right|^{2} d z\right\} .
\end{aligned}
$$

Все слагаемые справа, за исключением двух последних интегралов, уже обработаны в (4.32). Мажорантой для интеграла, содержащего $\bar{f}^{0}$, служит величина $\mathscr{N}_{0}^{2}$ (см. (4.13)). Наконец, согласно (4.5) и (4.3)

$$
\begin{aligned}
\int_{\Delta(h)} \rho^{2}\left|\overline{\mathbf{f}}^{1}\right|^{2} d z \leqslant c h^{2} \int_{\Delta(h)}\left|\overline{\mathbf{f}}^{1}\right|^{2} d z \leqslant c h^{2} \int_{\Delta(h)}\left(\sum_{i=1}^{2}\left|\mathscr{F}_{i}^{1}\right|^{2}\right. \\
\left.+h^{-2}\left\|\left(f_{3}^{0}, g_{3}^{0}\right) ; L_{2}(\omega(z)) \times L_{2}(\partial \omega(z))\right\|^{2}\right) d z \leqslant c\left(h^{2} \Sigma+\mathscr{N}_{0}^{2}\right),
\end{aligned}
$$


т.е. ввиду (4.32), (4.22) и (4.23) справедливость (4.30) сомнений не вызывает.

Осталось удостовериться в (4.29). Векторы $\mathbf{F}$ и $\mathbf{G}$ определены в (4.26). Обратим внимание на следуюшие факты: во-первых, $N(h, x)=1+O\left(h^{2}\right)$ (см. комментарий к (2.2)) и, во-вторых, в силу $(2.7),(4.4)$ и (4.6)

$$
\left((1-\chi) \mathbf{f}^{0}+\mathbf{f}^{1}, e^{i}\right)_{\omega(z)}+\left((1-\chi) g^{0}, e^{i}\right)_{\partial \omega(z)}=0, \quad i=1,2 .
$$

Следовательно, помятуя о структуре нормы (3.4) и применяя неравенства (3.30), (4.33) и (4.34), получаем

$$
\begin{aligned}
\mid(\mathbf{F}, \mathbf{R})_{\Omega_{h}} & +(\mathbf{G}, \mathbf{R})_{\Gamma_{h}} \mid \leqslant \\
\leqslant & c|\mathbf{R}|\left\{h ^ { 2 } \int _ { \Delta ( h ) } \left(h^{-2} \rho^{2}\left\|(1-\chi)\left(f^{0}, g^{0}\right) ; L_{2}(\omega(z)) \times L_{2}(\partial \omega(z))\right\|^{2}\right.\right. \\
& \left.+h^{-2} \rho^{2}\left\|\mathbf{f}^{1} ; L_{2}(\omega(z))\right\|^{2}+h^{-2} \rho^{4}\left|(1-\chi) \bar{f}^{0}+\overline{\mathbf{f}}^{1}\right|^{2}\right) d z \\
& \left.+h^{4} \int_{\Gamma_{h}} h^{-3} \rho^{4}\left|\mathbf{G}^{0}+\chi g^{0}\right|^{2} d s_{x}\right\} .
\end{aligned}
$$

В интегралах по $\Delta(h)$ вес $\rho$ безболезненно превращается в $h$ - таким образом возникают интегралы, которыми мы уже занимались в $(4.21),(4.35)$ и (4.36). За счет запасного множителя $h^{4}$ (фрагмент $1-N^{-1 / 2}$ в (4.26)) интеграл по $\Gamma_{h}$ также сводится к исследованным ранее интегралам. Значит, (4.29) действительно имеет место.

Сформулируем оценку, вытекаюшую из установленного неравенства (4.28).

Теорема 4.3. При сформулированных предположениях решение и задачи (1.6)-(1.8) и вспомогательное асимптотическое решение $\mathbf{U}$ удовлетворяют оченке

$$
|u-\mathbf{U}|+\left\|\varepsilon(u-\mathbf{U}) ; L_{2}\left(\Omega_{h}\right)\right\|^{2} \leqslant c_{\delta} h^{(1-\delta) / 2}\left(\mathscr{N}_{0}+\widetilde{\mathscr{N}_{\delta}}\right),
$$

где $\mathscr{N}_{0}$ и $\widetilde{N}_{\delta}$ - нормы слагаемых в представлении (2.6) (см. (4.13) и (4.24)), а постоянная $c_{\delta}$ зависит от $\delta>0$, но не от решения и и параметра $h \in(0,1)$.

4. Оправдание асимптотического представления решения. Аналогично выводу оценки (4.15) нетрудно проверить неравенство

$$
\left\|\varepsilon(u-\mathbf{U}) ; L_{2}\left(\Omega_{h}\right)\right\|^{2} \leqslant C_{\delta} h^{(1-\delta) / 2} \mathscr{N}_{0}
$$

Кроме того, прямые вычисления убеждают в том, что

$$
\left|h \mathscr{U}^{(1)}\right|+\left\|\varepsilon\left(h \mathscr{U}^{(1)}\right) ; L_{2}\left(\Omega_{h}\right)\right\| \leqslant c h^{1} \mathscr{N}_{0}
$$

(показатель степени $h$ в мажоранте предопределен величинами $h \| \partial \mathscr{U}_{i}^{(1)} / \partial y_{i}$; $L_{2}\left(\Omega_{h}\right)\|=\| \partial \mathscr{U}_{i}^{(1)} / \partial \eta_{i} ; L_{2}\left(\Omega_{h}\right) \|, i=1,2$, присутствующими в обоих слагаемых слева). Теперь предложение 4.1 и теорема 4.3 приводят к центральному утверждению статьи. 
ТЕОРема 4.4. При сформулированных предположениях (см. первые пункты в $\S 1-\S 3$ и п. 2 данного параграфа) решение и задачи (1.6)-(1.8) и асимптотическое решение $\mathscr{W}$ из (3.6), построенное в согласии с (2.16), (2.20), (2.25) u (2.36), (2.38), связаны соотношением

$$
|u-\mathscr{W}|+\left\|\varepsilon(u-\mathscr{W}) ; L_{2}\left(\Omega_{h}\right)\right\|^{2} \leqslant c_{\delta} h^{(1-\delta) / 2}\left(\mathscr{N}_{0}+\widetilde{\mathscr{N}}_{\delta}\right),
$$

где обозначения те же, что и в (4.37).

Так как $|\mathscr{W}|=O\left(h^{0}\right)$ (см. п. $\left.2 \S 3\right)$ и $\left\|\varepsilon(\mathscr{W}) ; L_{2}\left(\Omega_{h}\right)\right\|=O\left(h^{0}\right)$ (проверяется аналогично), теорема 4.4 в самом деле обосновывает асимптотику $u \sim \mathscr{W}$. Интегральная оценка (4.38) позволяет, в частности, установить сходимость функций $\Omega_{1} \ni(\eta, z) \mapsto h^{2} u_{i}(h \eta, z), i=1,2$ (нормированных поперечных смешений), в $L_{2}\left(\Omega_{1}\right)$ к компонентам $w_{i}$ решения $w$ результируюшей задачи $(2.36),(2.38)$. Более того, вьполняется неравенство

$$
\left\|h^{2} u_{i}-w_{i} ; L_{2}\left(\Omega_{1}\right)\right\| \leqslant c_{1} h\left(\mathscr{N}_{0}+\widetilde{\mathscr{N}}_{1}\right)
$$

Подчеркнем, что скорость сходимости ограничена величиной $C h$ из-за присутствия младших членов асимптотики $u_{i}$, содержаших $w_{4}$, а не мажорантой в (4.38), которую можно взять равной $c_{1} h^{0}\left(\mathscr{N}_{0}+\tilde{\mathscr{N}}_{1}\right)$ (дополнительный множитель $h$ появляется благодаря растяжению координат). Эти же члены препятствуют сходимости $u_{i}$ к $w_{i}$ в $H^{1}\left(\Omega_{1}\right)$.

После удаления из $u_{3}$ асимптотического фрагмента $(2.20)$, содержашего $w_{1}$ и $w_{2}$, получается такая оценка:

$$
\left\|h\left\{u_{3}+h^{-1}\left(\eta_{1} \partial_{z} w_{1}+\eta_{2} \partial_{z} w_{2}\right)\right\}-w_{3} ; L_{2}\left(\Omega_{1}\right)\right\| \leqslant c_{1} h\left(\mathscr{N}_{0}+\widetilde{\mathscr{N}}_{\delta}\right) .
$$

Для идентификации угла закручивания положим $u^{\prime}=\left(u_{1}, u_{2}\right)^{t}$ и

$$
\tilde{v}(y, z)=v(y, z)-|\omega(z)|^{-1} \int_{\omega(z)} v(h \eta, z) d \eta
$$

Теперь, используя проинтегрированное по $z \in(-1,1)$ неравенство Пуанкаре на сечении $\omega_{h}(z)$

$$
\left\|\rho^{-1} \tilde{v} ; L_{2}\left(\Omega_{h}\right)\right\| \leqslant \operatorname{ch}\left\|\rho^{-1} \nabla_{y} v ; L_{2}\left(\Omega_{h}\right)\right\|
$$

и вспоминая определение (3.4) нормы | | , получаем

$$
\left\|h \tilde{u}^{\prime}-\alpha\left(\tilde{\eta}_{1} e^{2 \prime}-\tilde{\eta}_{2} e^{1 \prime}\right) w_{4} ; L_{2}\left(\Omega_{1}\right)\right\| \leqslant \operatorname{ch}\left(\mathscr{N}_{0}+\widetilde{\mathscr{N}}_{\delta}\right) .
$$

Для деформации $\varepsilon(u)$ (а потому и для напряжений $\sigma(u)=A \varepsilon(u)$ ) на основании оценки (4.38) и сказанного в п. $2 \S 3$ выводим неравенство

$$
\left\|\varepsilon(u)-h^{-1}\left(\mathscr{Y}+\mathbb{D}_{\eta} \mathscr{V}\right) \mathscr{D}\left(\partial_{z}\right) w ; L_{2}\left(\Omega_{h}\right)\right\| \leqslant c_{\delta} h^{(1-\delta) / 2}\left(\mathscr{N}_{0}+\widetilde{\mathscr{N}}_{\delta}\right) .
$$


Отметим, что вычитаемое под знаком нормы является ничем иным, как столбцом деформаций, найденным согласно одномерной модели упругого стержня. Наконец, из приведенных формул вытекает соотношение, связывающее энергетические функционалы (2.43) и (2.24),

$$
|P(u, f, g)-\mathbf{P}(w, \mathscr{F})| \leqslant c_{\delta} h^{(1-\delta) / 2}\left(\mathscr{N}_{0}+\widetilde{N}_{\delta}\right)^{2} .
$$

Асимптотическая точность оценки (4.38) подтверждается известными исследованиями явления пограничного слоя вблизи торцов прямолинейного стержня (см. [23]-[27] и др.). Так, для компенсации невязки составляющей $h^{0} \mathscr{U}^{(0)}(\eta, z)$ в условиях Дирихле (1.8) приходится в асимптотическое разложение (2.9) добавить слагаемые $h^{0} \mathscr{X}^{ \pm}\left(\xi^{ \pm}\right)$, записанные в быстрых переменных $\xi^{ \pm}=\left(h^{-1} y, h^{-1}(1 \mp z)\right)$ и являюшиеся экспоненциально затухаюшими на бесконечности решениями специальных задач теории упругости в полуцилиндрах $\omega( \pm 1) \times \mathbb{R}_{+}$. Вычисляя нормы $\left|\mathscr{X}^{ \pm}\right|$и $\left\|\varepsilon\left(\mathscr{X}^{ \pm}\right) ; L_{2}\left(\Omega_{h}\right)\right\|$, видим, что они равны $O\left(h^{1 / 2}\right)$. Таким образом, из-за отсутствия $\mathscr{X}^{ \pm}$в асимптотическом решении $\mathscr{W}$ показатель степени $h$ в оценке (4.38) нельзя сделать большим $1 / 2$, что и находит свое отражение в неравенстве $\delta>0$. Кроме того, величины $\varepsilon\left(\mathscr{X}^{ \pm}\right)$и $\sigma\left(\mathscr{X}^{ \pm}\right)$имеют вблизи торцов стержня $\Omega_{h}$ тот же порядок $h^{-1}$, что $\varepsilon(\mathscr{W})$ и $\sigma(\mathscr{W})$, т.е. рассчитывать на равномерное приближение пространственных полей деформаций и напряжений асимптотическими конструкциями (3.6) или (2.9) не приходится, а интегральная оценка (4.40) стала возможной только благодаря экспоненциальному затуханию пограничного слоя на удалении от торцов стержня.

ЗАмечание 4.5. Согласно (2.16), (2.20) и $(2.25)$ включение $\mathscr{W} \in H^{1}\left(\Omega_{h}\right)^{3}$ обеспечивается условием $w \in H^{3}(-1,1)^{2} \times H^{2}(-1,1)^{2}$, более слабым, чем указанное в (4.14) (ранее в приближенном решении $\mathscr{U}$ использовалось слагаемое $h^{1} \mathscr{U}^{(1)}$, требовавшее двукратной дифференцируемости $\left.\mathscr{D}\left(\partial_{z}\right) w(z)\right)$. Это обстоятельство позволяет сформулировать гипотезу: теорема 4.4 остается верной и при уменьшении на единицу показателей гладкости по переменной $z$ в предположении (4.13). Вопрос о ее справедливости, ответить на которьй при помощи изложенного в статье подхода, к сожалению, не удалось, весьма важен для приложений, поскольку расширяет область обоснованной применимости одномерных моделей. Например, условия (4.13) отвергает кусочно постоянное погружение вдоль оси цилиндра; в то же время нормы $|\cdot|$ и ||$\varepsilon(\cdot) ; L_{2}\left(\Omega_{h}\right)||$ дополнительного внутреннего пограничного слоя, возникаюшего вблизи поверхности $\left\{z=z^{0}\right\}$ разрывов функций $f^{0}, g^{0}$ и описываемого решениями задачи теории упругости в цилиндре $\omega\left(z^{0}\right) \times \mathbb{R}$, оказываются величинами $O(h)$ и не могут повлиять на мажоранту в (4.38).

\section{Список литературы}

1. Clebsch A. Theorie der Elastizität der festen Körper. Leipzig, 1862.

2. Тимошенко С. П. Колебания в инженерном деле. М.: Наука, 1967.

3. Лурье Ю.Н., Джанелидзе Г.Ю. Задача Сан-Венана для естественно скрученных стержней // ДАН СССР. 1939. Т. 24. № 1. С. 23-26; № 3. С. 225-229; ㄲo4. С. 325-326.

4. Лехницкий С. Г. Кручение анизотропных и неоднородных стержней. М.: Наука, 1971. 
5. Работнов Ю. Н. Механика деформируемого твердого тела. М.: Наука, 1978.

6. Илюхин A. A. Пространственные задачи нелинейной теории упругих стержней. Киев: Наукова думка, 1979.

7. Ржаницьн А.Р. Строительная механика. М.: Высшая школа, 1982.

8. Светличкий В. А. Механика стержней. Т. 1, 2. М.: Высшая школа, 1987.

9. Бердичевский В. Л. Об энергии упругого стержня // Прикладная математика и механика. 1981. Т. 40. № 4. С. 704-718.

10. Елисеев B. В. К нелинейной динамике упругого стержня // Прикладная математика и механика. 1988. Т. 52. №4. С. $635-642$.

11. Галактионов E. B., Tponn Э.А. Асимптотический метод расчета термоупругих напряжений в тонком стержне // Изв. АН СССР. Сер. физ. 1976. Т. 40. № 7. С. 1399-1406.

12. Sanchez-Hubert J., Sanchez-Palencia E. Couplage flexion-torsion-traction dans les poutres anisotropes a section heterogene // C. R. Acad. Sci. Paris. Ser. 2. 1991. V. 312. P. 337-344.

13. Sanchez-Hubert J., Sanchez-Palencia E. Coques elastiques mines. Proprietes asymptotiques. Paris: Masson, 1997.

14. Елисеев В. В., Орлов С.Г. Асимптотическое расщепление в пространственной задаче линейной упругости для удлиненных тел со структурой // Прикладная математика и механика. 1999. Т. 63. № 1. С. 93-101.

15. Шойхет Б. А. Об асимптотически точных уравнениях тонких плит сложной структуры // Прикладная математика и механика. 1973. Т. 37. № 5. С. 914-924.

16. Ciarlet P. C., Destuynder P. A. Justification of the two-dimensional linear plate model // J. Mecanique. 1979. V. 18. P. 315-344.

17. Bermúdez A., Viaño J.M. Une justification des équations de la thermoélasticité des poutres à section variable par des méthodes asymptotiques // RAIRO Analyce Numérique. 1984. V. 18. P. 347-376.

18. Tutek Z., Aganovich I. A justification of the one-dimensional model of an elastic beam // Math. Methods in Appl. Sci. 1986. V. 8. P. 1-14.

19. Trabucho de Campos L., Viaño J. M. Existence and characterization of higher order terms in an asymptotic expansion method for linearized elastic beams // Asymptotic Analysis. 1989. V. 2. P. 223-255.

20. Le Dret H. Modeling of the junction between two rods // J. Math. Pures Appl. 1989. V. 68. P. 365-397.

21. Le Dret $H$. Problemes variationnels dans les multi-domains modélisation des jonctions et applications. Paris: Masson, 1991.

22. Veiga M.F. Asymptotic method applied to a beam with a variable cross section // Asymptotic methods for elastic Structures. Berlin-N.Y.: Walter de Gruyter, 1995. P. 237-254.

23. Назаров С. А. Структура решения эллиптических краевых задач и тонких областях // Вестн. ЛГУ. 1982. № 7. С. 65-68.

24. Козлова M. В. Осреднение трехмерной задачи теории упругости для тонкого неоднородного бруса // Вестн. МГУ. Сер. матем., мех. 1989. № 5. Р. 6-10.

25. Козлова М. В., Панасенко Г. П. Осреднение трехмерной задачи теории упругости в неоднородном стержне // ЖВМиМФ. 1991. Т. 31. № 10. С. 1592-1596.

26. Panasenko G.P. Asymptotic analysis of bar systems. I // Russian J. of Math. Physics. 1994. V. 2. № 3. P. 325-352; II // ibid. 1996. V. 4. № 1. P. 87-116.

27. Mazja W. G., Nasarow S.A., Plamenewskij B.A. Asymptotische Theorie elliptischer Randwertaufgaben in singulär gestörten Gebieten. Bd. 2. Berlin: Academie-Verlag, 1991.

28. Леора C. Н., Назаров С. А., Проскура A. В. Вывод предельных уравнений для эллиптических задач в тонких областях при помощи ЭВМ // ЖВМиМФ. 1986. Т. 26. № 7 . C. $1032-1048$. 
29. Назаров C. A. Общая схема осреднения самосопряженных эллиптических систем в многомерных областях, в том числе тонких // Алгебра и анализ. 1995. Т. 7. № 5. С. 1-92.

30. Назаров C.A., Пилецкас К.И. Рейнольдсово течение жидкости в тонком трехмерном канале // Литовский матем. сб. 1990. Т. 30. №4. С. $772-783$.

31. Kondratiev V.A., Oleinik O.A. Hardy's and Korn's type inequalities and their applications // Rendiconti di Matematica. Ser. VII. 1990. V. 10. P. 641-666.

32. Назаров C. А. Неравенства Корна, асимптотически точные для тонких областей // Вестн. СПбГУ. 1992. № 8. Р. 19-24.

33. Cioranecu D., Oleinik O.A., Tronel G. Korn's inequalitites for frame type structures and junctions with sharp estimates for the constants // Asymptotic analysis. 1994. V. 8. P. 1-14.

34. Nazarov S. A. Korn's inequalities for junctions of spatial bodies and thin rods // Math. Meth. Appl. Sci. 1997. V. 20. № 3. P. 219-243.

35. Назаров C.A. Самосопряженные эллиптические краевые задачи. Полиноминальное свойство и формально положительные операторы // Проблемы матем. анализа. Вып. 16. СПб: Изд-во СПбГУ, 1997. С. 167-192.

36. Кондратьев B. А., Олейник О.А. О зависимости констант в неравенстве Корна от параметра, характеризующего геометрию области // УМН. 1989. Т. 44. №6. С. 157-158.

37. Кондратьев B.A., Олейник О.А. Краевые задачи для системы теории упругости в неограниченных областях. Неравенство Корна // УМН. 1988. Т. 43. № 5. С. 55-98.

38. Назаров C. A. Обоснование асимптотической теории тонких стержней. Интегральные и поточечные оценки // Проблемы матем. анализа. Вып. 17. СПб: Изд-во СПбГУ, 1997. P. 101-152.

39. Харди Г. Г., Литтлвуд Дж. Е., Полиа Г. Неравенства. М.: ИЛ, 1948.

Поступило в редакцию

3.VIII. 1998 